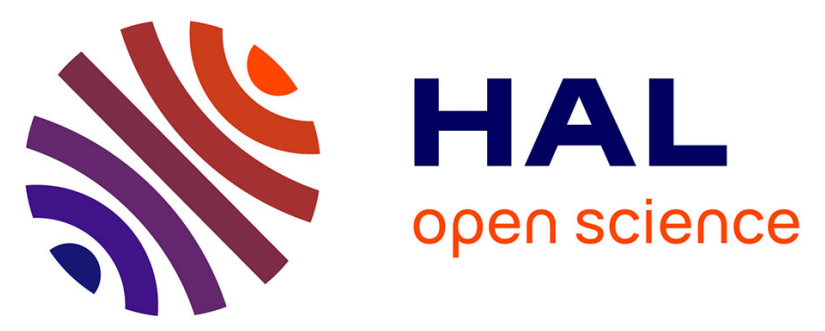

\title{
Brive-la-Gaillarde (Corrèze). Les sarcophages de la nécropole Saint-Martin de Brive: particularités et aménagements, fin Ve siècle-VIIIe siècle
}

Emmanuel Barbier, Guillaume Rougé, Pauline Duneufjardin, Katia Lagorsse

\section{- To cite this version:}

Emmanuel Barbier, Guillaume Rougé, Pauline Duneufjardin, Katia Lagorsse. Brive-la-Gaillarde (Corrèze). Les sarcophages de la nécropole Saint-Martin de Brive: particularités et aménagements, fin Ve siècle-VIIIe siècle. Archéologie du Midi Médiéval, 2017, 35, pp.241-262. 10.3406/amime.2017.2155 . halshs-02497464

\author{
HAL Id: halshs-02497464 \\ https://shs.hal.science/halshs-02497464
}

Submitted on 3 Mar 2020

HAL is a multi-disciplinary open access archive for the deposit and dissemination of scientific research documents, whether they are published or not. The documents may come from teaching and research institutions in France or abroad, or from public or private research centers.
L'archive ouverte pluridisciplinaire HAL, est destinée au dépôt et à la diffusion de documents scientifiques de niveau recherche, publiés ou non, émanant des établissements d'enseignement et de recherche français ou étrangers, des laboratoires publics ou privés. 


\section{Brive-la-Gaillarde (Corrèze). Les sarcophages de la nécropole} Saint-Martin de Brive : particularités et aménagements, fin Ve siècle-VIIIe siècle

Emmanuel Barbier, Guillaume Rougé, Pauline Duneufjardin, Katia Lagorsse

\section{Citer ce document / Cite this document :}

Barbier Emmanuel, Rougé Guillaume, Duneufjardin Pauline, Lagorsse Katia. Brive-la-Gaillarde (Corrèze). Les sarcophages de la nécropole Saint-Martin de Brive : particularités et aménagements, fin Ve siècle-VIIle siècle. In: Archéologie du Midi médiéval. Tome 35, 2017. pp. 241-262;

doi : https://doi.org/10.3406/amime.2017.2155

https://www.persee.fr/doc/amime_0758-7708_2017_num_35_1_2155

Fichier pdf généré le 06/01/2020 
NOUVELLE AQUITAINE

BRIVE-LA-GAILLARDE (CORRÈZE)

\section{LES SARCOPHAGES DE LA NÉCROPOLE SAINT-MARTIN DE BRIVE : PARTICULARITÉS ET AMÉNAGEMENTS (FIN V' ${ }^{\mathrm{e}}$ SIÈCLE-VIII' ${ }^{\mathrm{e}}$ SIÈCLE)}

\author{
Emmanuel BARBIER*, \\ Guillaume ROUGÉ ** \\ avec la collaboration de \\ Pauline DUNEUFJARDIN*** \\ et de Katia LAGORSSE****
}

Le cœur de la ville de Brive (Corrèze) a fait l'objet, au cours des années 2010, d'un vaste programme de rénovation urbaine. Il consistait en une reprise du revêtement et des réseaux complétée par un aménagement paysager (plantation d'arbres, fontaines). Ce projet se développant autour de l'actuelle église paroissiale SaintMartin, siège de l'ancienne collégiale éponyme, une fouille des abords a pu être effectuée en 2012.

Celle-ci s'est limitée aux secteurs les plus impactés par le projet (fig. 1). La cote de fond de fouille n'excédait pas $0,80 \mathrm{~m}$ à l'exception de zones balisées (plantations, reprises de réseaux). Cette modalité n'a pas permis d'appréhender, au sein de nombreux secteurs, les premières formes d'occupation, notamment celles liées au développement de la nécropole. L'objectif de cette étude est de présenter l'évolution de la topographie funéraire depuis la fin $\mathrm{du}^{\mathrm{e}}$ siècle jusqu'au VIII' ${ }^{\circ}$ siècle. Les données relatives aux architectures seront privilégiées. Dans ce cadre, l'étude biologique des différents individus inhumés ne sera pas détaillée.

\section{BRIVE : UN PEUPLEMENT MÉCONNU}

\section{Une situation «stratégique»}

La ville de Brive est située à la limite des zones métamorphiques du Massif Central et des zones sédimentaires du Bassin Aquitain. Elle se développe au sein d'un bassin sédimentaire essentiellement composé de roches sédimentaires communément qualifiées de " grès ».

La ville s'est développée sur une dorsale topographique de $112 \mathrm{~m}$ d'altitude moyenne constituée par des alluvions du quaternaire récent. Celle-ci est limitée tant au nord qu'au sud par des reliefs escarpés composés de grès primaires. La rivière Corrèze, qui prend sa source sur le plateau de Millevaches, traverse ce territoire d'est en ouest. Si l'opportunité d'un franchissement de la Corrèze sur les marges occidentales du Massif Central a participé au développement de l'agglomération de Brive, d'importantes contraintes persistaient de par cette position au sein d'une terrasse alluviale. Dans ce contexte, les premières formes d'occupation ont privilégié les faibles reliefs culminant à $115 \mathrm{~m}$ au détriment des berges méridionales de la terrasse bordant la Corrèze situées à 110 m NGF. Deux légères éminences, les puys SaintPierre et Saint-Martin, séparées par un affluent de la Corrèze, le Verdanson, se détachent en effet de cet ensemble.

Les conditions propices au développement de la ville relèvent donc de cette position de carrefour au point de franchissement d'un cours d'eau capricieux : la Corrèze.

$\mathrm{Au}$ regard de cet environnement quelque peu hostile, les premières formes d'occupation paraissent relativement tardives. Les données issues de diverses opérations archéologiques suggèrent une occupation exclusivement antique (Ir siècle) et principalement située à l'est de la collégiale depuis le Puy Saint-Pierre jusqu'à la place Massénat (Roger 2004) située à moins de $200 \mathrm{~m}$ du chevet (fig. 2). En dépit de découvertes fortuites, notamment au chevet de l'église (Moser et al., 1978, 101-102), elle ne semble pas s'étendre jusqu'à la collégiale. Elle se manifeste sous la forme de petits habitats et d'ateliers desservis par deux voiries principales dont le tracé est encore perceptible dans le parcellaire viaire (fig. 2).

Ces voiries ont conditionné le développement de la ville et l'installation des premières églises Saint-Pierre, Saint-Sernin et Saint Martin. Le développement du culte de Martin est essentiellement renseigné par les écrits de Grégoire de Tours (mort en 594) qui fait état d'un culte de saint Martin dit " l'Espagnol » dès le $\mathrm{VI}^{\mathrm{e}}$ siècle (Proust, 2005, 243) (1). Il nous apprend qu'en 407, un jeune disciple de saint Martin de Tours du nom de Martin l'Espagnol, arrive dans le vicus de Brive et saccage le temple païen. Cet acte est sanctionné par une lapidation et une mort à la gloire du martyr. Au-delà du caractère hagiographique du récit récemment commenté par Anne Massoni (Massoni 2012, 108), il importe toutefois de considérer la contemporanéité entre saint Martin de Brive et l'évangélisateur éponyme de Tours qui meurt en 397. Le décès du premier intervient probablement au

\footnotetext{
* Responsable de recherche archéologique, Inrap, membre associé UMR 7302 (Centre d’Études Supérieures et de Civilisation Médiévale, Université de Poitiers).

** Docteur, Université Bordeaux-Montaigne, UMR 5607 Ausonius.

*** Anthropologue, Inrap.

**** Responsable de secteur, Inrap.

${ }^{1}$ Gregorii Episcopi Turonensis Historiarum, Liber VII, 10 in Krusch B., Levinson W. (ed.), Monumenta Germaniae Historica, Scriptores Rerum

Merovingicarum, T. I, 1951, p. 332: « ...Briva Curretia vicum, in quo sanctus Martinus, nostri ut aiunt Martini discipulus, requiescit... ».
} 


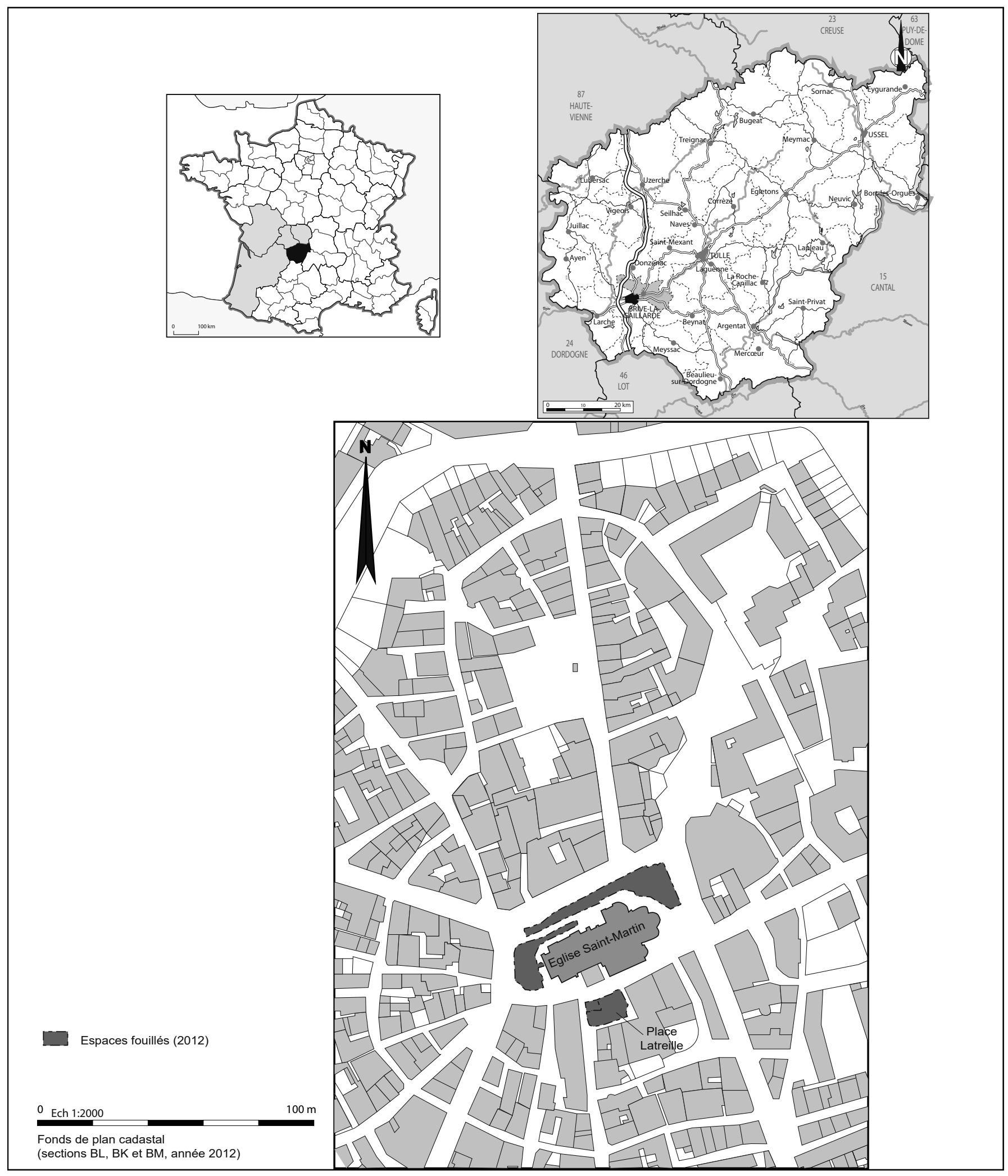

Fig. 1 : Localisation du site et de l'emprise fouillée (E. Barbier, Inrap). 


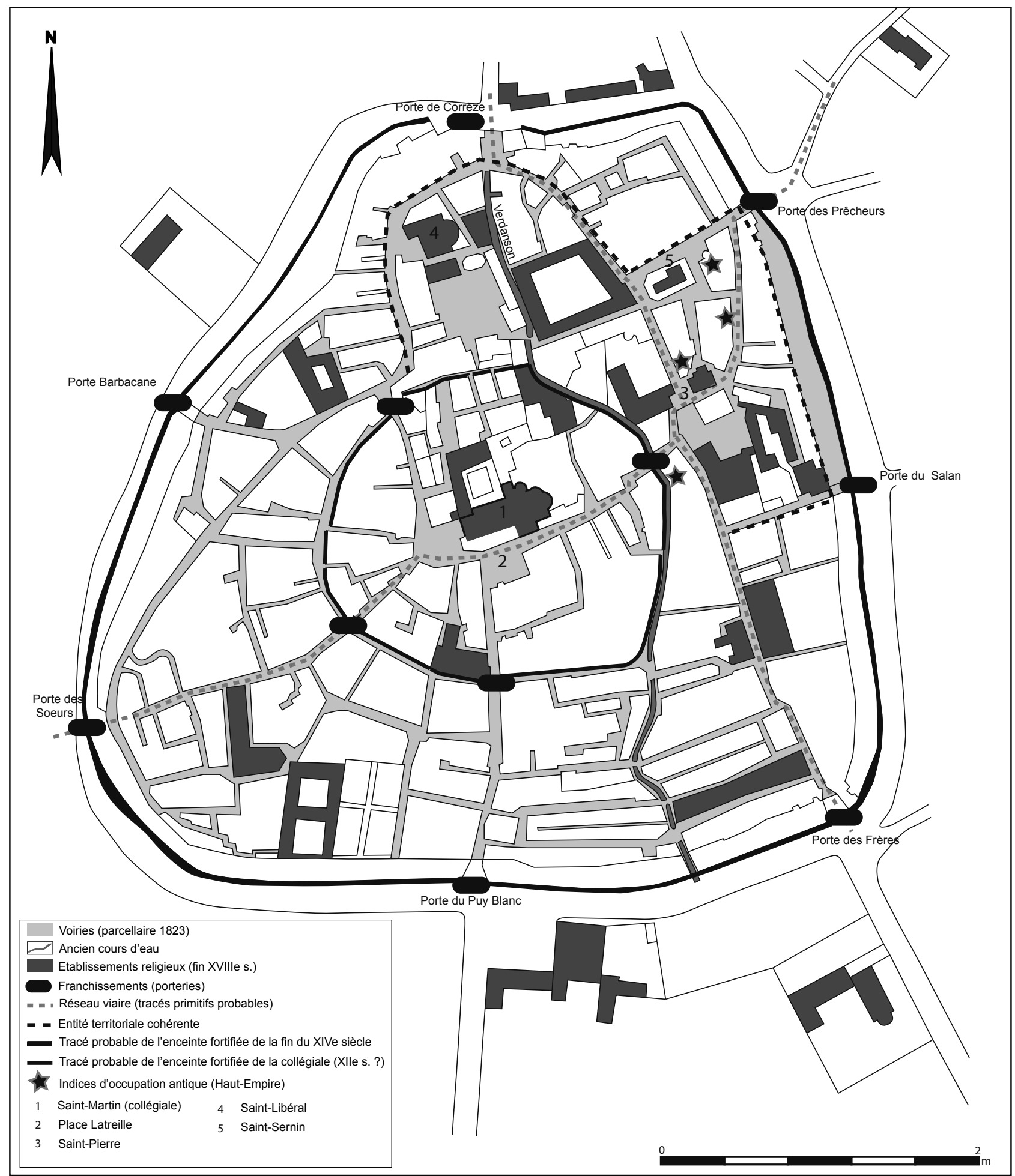

Fig. 2 : Axes «structurants» attestés au sein de la ville de Brive (DAO : B. Oliveau, E. Barbier, Inrap. Fonds de plan : Chavent, 2006).

cours du $\mathrm{IV}^{\mathrm{e}}$ siècle. Il aurait été inhumé à l'écart du Puy Saint-Pierre où semblait se concentrer l'occupation pour la période antique. Son inhumation suscite un intérêt certain et vraisemblablement immédiat qui pourrait avoir précédé l'épiscopat de l'évêque de Limoges Rurice I ${ }^{\text {er }}$ (485-507) réputé pour avoir organisé le culte autour du tombeau de
Martin (Massoni 2012, 107). La conservation d'une correspondance entre l'évêque de Limoges et le prélat Clarus d'Eauze semble le confirmer. Ce dernier y est remercié pour l'envoi de colonnes auquel s'ajoute la commande de dix autres (Massoni, 2012) (2) démontrant ainsi la réalisation de travaux en relation avec le culte de Martin. 
Grégoire de Tours évoque la basilique récemment reconstruite par l'évêque de Limoges Ferréol suite à l'incendie (3) survenu en 584 « à tel point que l'autel et les colonnes qui étaient de différentes espèces de marbre furent réduits en cendre $»$. Un nouvel édifice paraît donc élevé à la fin du $\mathrm{VI}^{\mathrm{e}}$ siècle.

Le soin manifesté par l'évêque de Limoges démontre l'intérêt suscité pour ces territoires situés sur les marges du diocèse. L'entretien du culte de Martin participe tant de l'encadrement d'une population que d'une mainmise politique sur un espace situé à un véritable carrefour de voies menant de Limoges à Toulouse et de Clermont à Bordeaux.

Ce culte de Martin est attesté au cours du VII ${ }^{e}$ siècle étant donné son évocation dans le martyrologe dit hyéronimien puis dans celui d'Usuard rédigé vers 865 (Massoni 2012, 108). Ces documents ne nous renseignent pas sur son organisation. Son ampleur et la présence de personnel (clercs, prêtres) dédié à son entretien demeurent méconnues.

\section{Les données archéologiques}

La collégiale Saint-Martin a suscité l'intérêt de nombreux archéologues depuis la fin du $\mathrm{XIX}^{\mathrm{e}}$ siècle et les premiers travaux entrepris sur l'édifice.

Dès 1877, des sépultures en sarcophages rectangulaires furent découvertes en amont des travaux de construction du porche (fig. 3). Si les derniers niveaux de sépultures en cercueil apparaissaient à un mètre sous le niveau de sol (Bonnay 1879, 225), des inhumations en coffrages « en pierre trapézoïdales avec logettes céphaliques de pierre de Gramont » étaient installées sur un premier niveau de sépultures en sarcophages (Bonnay 1879, 230). De même, de nombreux éléments architecturaux (fragments de tegulae) dont certains relevant d'une construction de «prestige » furent dégagés (base de colonne dorique en marbre). Les travaux du porche réalisés en 1878 confirmèrent l'occupation funéraire de cet espace. Les plans fournis par l'archéologue permettent d'identifier près d'une vingtaine de sépultures en sarcophages « en pierre de brasier» (grès local). Le couvercle « monolithe, légèrement bombé, est quelquefois à deux pentes ; l'auge, également d'une seule pièce, a une place arrondie pour recevoir la tête » (Bonnay 1879, 230). Les dimensions renseignées varient pour la seule longueur entre 2,23 m et $0,80 \mathrm{~m}$ suggérant la présence d'une population d'âges variés. Les cuves étaient perturbées par de précédentes interventions à l'exception d'une seule inhumation a priori préservée et couverte d'un « mélange de terre et de charbon » qui ne semble pas relever, selon l'auteur, d'une infiltration, mais d'un dépôt intentionnel.
Plus récemment, des opérations diverses réalisées aux abords de la collégiale ont permis d'appréhender l'ampleur de la nécropole qui semblait donc se poursuivre contre le mur gouttereau nord et au-delà du chevet roman (Audouy 1978, 102). Trois niveaux de sépultures ont été observés pour l'un des sondages qui présentait six tombes bâties. Des fragments de sarcophages monolithes étaient mêlés aux déblais qui comptaient par ailleurs quelques fragments erratiques de mobilier antique. Au nord de la collégiale, les perturbations la période Moderne avaient toutefois épargné deux inhumations en sarcophages, encore pourvue pour l'une d'entre elles d'un fragment de couvercle, ainsi que deux sépultures bâties (Audouy, 1978, 107-109). Ces vestiges sont apparus entre $1 \mathrm{~m}$ et 1,20 m sous le niveau de sol.

Ces premières données ont pu être complétées au cours de deux campagnes de fouilles plus récentes réalisées en 1986 à la croisée du transept de la collégiale avant d'être poursuivies en janvier 1987 au niveau de la nef. Celles-ci ont révélé les vestiges d'un bâtiment rectangulaire $(5 \mathrm{~m} \times 11 \mathrm{~m})$. Orienté $\mathrm{E} / \mathrm{O}$, il est interprété comme un petit sanctuaire ou mausolée à chevet plat qui semble enserrer des sépultures en sarcophages (fig. 3). Trois étapes de construction ont alors été distinguées (Cantié G. 1987, 69). La première a été identifiée par les traces de rubéfaction visibles sur les vestiges des murs M4 et M1 (4). Les murs liés au mortier de tuileau sont montés à l'aide de petits « moellons cubiques en grès de pays ». La tradition antique de la construction et les traces de rubéfaction permettraient, selon l'auteur, d'identifier cet ensemble comme l'édifice incendié qui est mentionné par Grégoire de Tours (cf. supra).

La seconde consiste en une reprise du bâtiment précédent sans en modifier le plan général. Les maçonneries mieux conservées sont assises sur une fondation en pierre de taille légèrement débordante complétée en partie inférieure par un blocage de gros galets. Les murs étaient vraisemblablement enduits et délimitaient un espace pourvu d'un niveau de sol en béton de tuileau (5) qui semblait sceller un premier niveau d'inhumations en sarcophages. Ces derniers sont taillés dans un matériau local (grès) et adoptent un plan trapézoïdal. Près de six sarcophages ont été observés au sein de cet espace. Trois d'entre eux étaient encore pourvus de leur couvercle. Ils sont installés dans un remblai composé de «cailloux, sable et tegulae » (Cantié 1987, 74). Cette reprise générale du bâtiment est interprétée comme la réhabilitation de l'édifice par l'évêque Férréol dans la seconde moitié du VI ${ }^{\mathrm{e}}$ siècle (cf. supra) (6).

\footnotetext{
${ }^{3}$ Grégoire de Tours, Historia Francorum, VII, 10 in Krusch B., Levinson W. (ed.), Momumenta Germaniae Historica, Scriptores Rerum Merovingicarum, T. I, 1951, p. 332 : « Magno ea tempestate incendio basilica antedicti Martini apud Brivam vicum ab imminente hoste cremata est (...) Sed ita aedes in posterum a Ferreolo episcopo reparata est, tamquam si nihil mali pertulerit... ».

${ }^{4}$ L'auteur présente ce vestige comme postérieur aux inhumations en sarcophages. Or, cette partie du site encore visible actuellement (crypte archéologique) fait état d'une relation. Dans ce cadre, le bâtiment ou sa reprise ( $2^{\text {nde }}$ phase) serait postérieur à la première phase d'inhumation en sarcophages. ${ }^{5}$ Ce niveau de sol est repris au cours d'une seconde phase d'aménagement de l'espace dès lors pourvu d'un sol composite. Ce dernier est composé de petits pavés de forme tronco-pyramidale tantôt en grès rouge tantôt en calcaire oolithique. L'ensemble est disposé selon un damier bicolore pris dans un béton de tuileau (Cantié G ? 1987, 76). Un niveau de sol similaire a été observé au sein de l'église Saint-Pierre de Brive (Labrousse M. 1955, 90). ${ }^{6}$ Des éléments lapidaires ont été dégagés dans les remblais de démolition qui accompagnaient ces structures : fragments de fûts de colonne en marbre et restes d'une inscription sur enduits attribués au VIII ${ }^{\mathrm{e}}$ siècle. Cette orientation chronologique pose question au regard des interprétations proposées par l'auteur qui attribue la reprise du bâtiment à l'évêque Ferréol (deuxième moitié $\mathrm{VI}^{\mathrm{e}}$ siècle).
} 


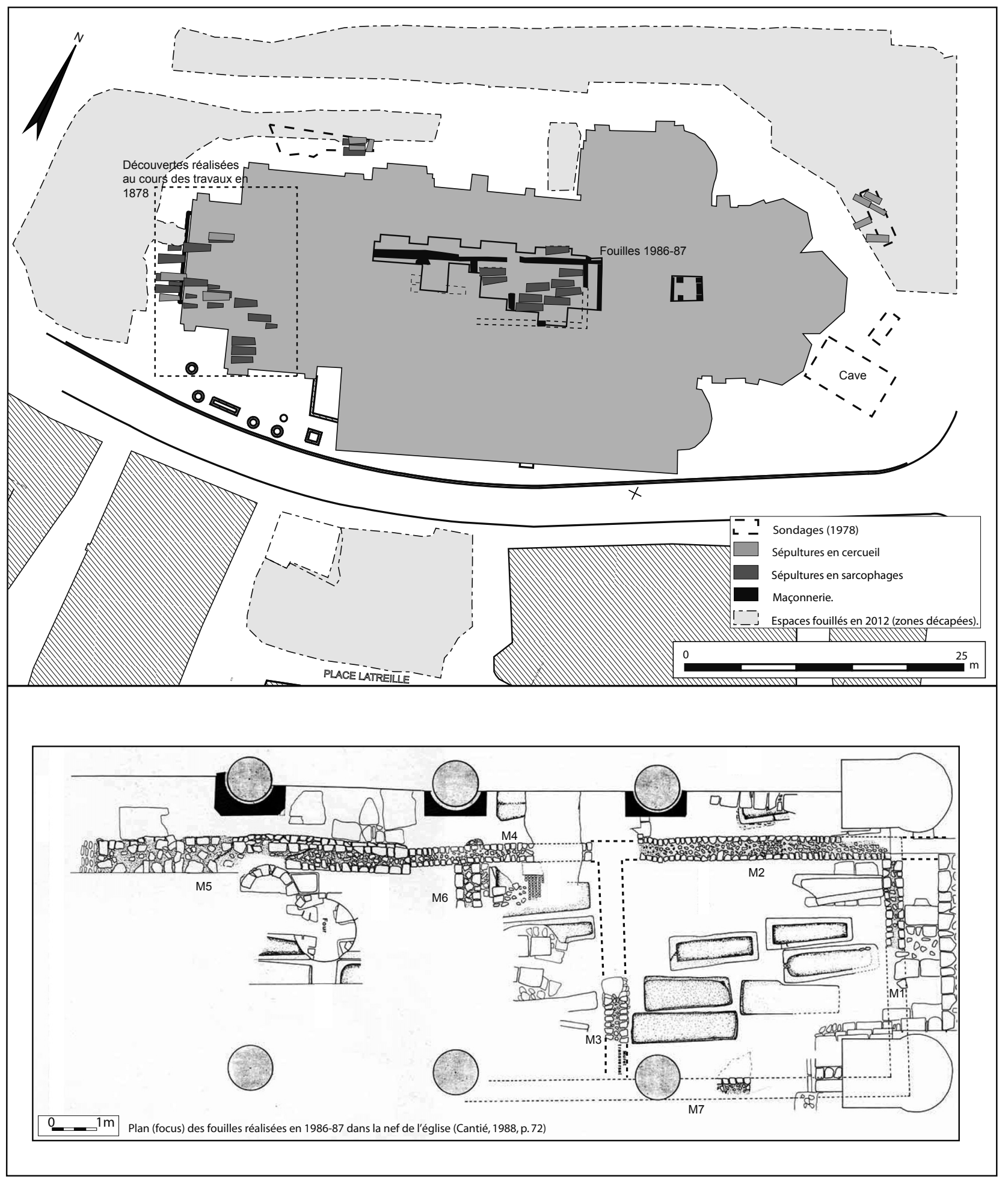

Fig. 3 : Vestiges découverts depuis la fin du XIX siècle au sein et aux abords de la collégiale (DAO : E. Barbier, F. Chevreuse, K. Lagorsse, Inrap).

Au cours d'une dernière phase d'aménagement, une nouvelle maçonnerie (M5) est construite. Elle prolonge d'au moins $7 \mathrm{~m}$ le bâtiment vers l'ouest. Sa mise en œuvre est différente de celles adoptées pour les précédentes constructions. Les moellons sont plus grands et mêlés à des fragments de sarcophages, le tout étant désormais lié à un mortier de chaux de couleur blan- châtre. Une construction semi-circulaire, partiellement conservée suite à la construction d'un four, jouxte cette nouvelle maçonnerie. Elle est présentée par l'auteur comme un possible baptistère.

Par ailleurs, G. Cantié signale la réalisation d'un sondage complémentaire au niveau du chevet de la collégiale. Réalisé à $2 \mathrm{~m}$ de profondeur sous le niveau de sol 
actuel, il a permis de dégager une structure reposant sur un sol de mortier blanc. Celle-ci était délimitée par 4 colonnes réparties selon un plan quadrangulaire moyennant un espacement régulier entre chacune d'entre elles.

Si ces données récentes renouvellent considérablement les connaissances sur cette nécropole et confirment la présence de bâtiments en lien avec le culte de Martin, l'interprétation générale, largement inspirée par les faits historiques, néglige certains aspects. A cet égard, la présentation de la première phase d'aménagement repose uniquement sur l'observation des seules traces de rubéfaction. Or, l'observation du bâti démontre une chronologie relative inverse sur la partie orientale où le mur Est apparaît postérieur au mur gouttereau nord, pourtant attribué à une seconde phase d'aménagement. Ainsi, de nombreuses questions restent en suspens telles :

La relation étroite entre les murs de " refend » et les sarcophages qui ne sont pas précisées. Pourtant, leur construction respective conditionne la topographie funéraire, les cuves de sarcophages étant insérées au sein de trois espaces distincts strictement délimités par des ces murets.

Le phasage des inhumations n'est pas développé. Deux niveaux d'inhumation sont pourtant attestés, le second étant vraisemblablement associé avec une reprise des bâtiments pourvus de niveaux de sols maçonnés.

Si la présence d'une nécropole ad sanctos semblait très probable, son étendue, son organisation ainsi que sa conservation demeuraient méconnus. La chronologie était, par ailleurs, soumise à de nombreuses interprétations fortement influencées par le récit de Grégoire de Tours.

\section{LA TOPOGRAPHIE FUNÉRAIRE DEPUIS LE V' SIÈCLE JUSQU'A LA FIN DU VII' SIÈCLE}

Deux périodes d'inhumation distinctes rassemblant près de 125 tombes potentielles ont pu être mises en évidence sur l'ensemble du site. Les relations en chronologie relative documentées, en outre, par 15 datations issues d'analyses au radiocarbone ont déterminé cette distinction chronologique. Seul le chevet ne présente pas d'inhumation pour cette période, mais cette donnée mérite d'être nuancée au regard des informations issues d'une opération archéologique plus ancienne (1978) au cours de laquelle des fragments de sarcophages monolithes furent dégagés à près de $2 \mathrm{~m}$ sous le niveau de la place au contact du chevet de la collégiale (Moser et al. 1978, 102-103). Or, ces niveaux n'ont pas pu être atteints dans le cadre de l'opération (fig. 4). En définitive, le cadre de l'opération n'a pas permis d'appréhender l'ensemble du paysage funéraire pour ces deux périodes d'inhumation. Quelques secteurs ont bénéficié d'investigations plus approfondies qui autorisent toutefois une lecture assez précise de la topographie funéraire, notamment sur le parvis, au nord et au sud de l'église.

La majorité des sépultures dégagées sont installées au sein de sarcophages $(113 / 125)$ en grès local. Celles-ci se succèdent très rapidement comme semblent le confirmer les datations ${ }^{14} \mathrm{C}$ réalisées sur près de 15 sépultures. Quelques observations majeures et communes, pour certaines, à ces deux périodes peuvent être dégagées.

\section{La première période d'inhumations ( $V^{e}$ - début VI ${ }^{e}$ siècle)}

\section{La topographie funéraire de la première période d'inhumations}

Près de 49 inhumations ont été identifiées pour cette période : 46 au sein de sarcophages, une, au nord, en contenant rigide, et deux autres pour lesquels les contenants n'ont pas pu être déterminés. Les cuves sont toutes orientées est-ouest même si de très légères variations sont perceptibles au nord (fig. 5). Elles sont installées au contact des dépôts alluvionnaires qui ne présentent aucun vestige susceptible d'identifier une occupation antérieure. La qualité de conservation des différentes structures funéraires est relativement bonne à l'exception du secteur nord où l'installation a posteriori du jardin a contraint à un décaissement dont l'impact sur les contenants paraît très limité. Ainsi, les sarcophages sont, pour une grande majorité, encore pourvus de leur couvercle, scellé à la cuve pour certains.

Deux principaux ensembles ont toutefois été identifiés. Le premier se développe autour de l'actuelle église au contraire du second, situé au sud, et distant d'une dizaine de mètres du mur gouttereau sud.

$\mathrm{Au}$ sein du premier groupe, les sépultures s'organisent en rangées plus ou moins jointives et délimitées par des contraintes monumentales (maçonneries) ou plus suggestives (haies...etc). Cette observation vaut notamment pour les inhumations du parvis dont l'agencement général paraît limité tant à l'est qu'à l'ouest par deux constructions maçonnées (M128 et M133), sans se prolonger vers le sud en dépit d'une absence matérialisée de limite comme en atteste la réalisation d'un sondage profond. L'organisation des rangées semble variable parmi les différents secteurs observés. Les sépultures semblent en effet jointives au nord-ouest, interdisant toute possibilité de circulation entre les différents contenants. Au contraire, cette organisation paraît plus lâche et espacée par groupes restreints de 3 à 4 individus au nord puis relativement désordonnée à l'ouest.

Des niveaux de piétinement ou d'installation ont été repérés très localement aux abords des cuves des sarcophages, notamment au nord-ouest. Ceux-ci apparaissent de manière sporadique et non extensive et semblent associés aux sarcophages. Cette observation vaut notamment pour le sarcophage 3304 à coté duquel un épandage de mortier de chaux de couleur blanchâtre a été observé (fig. 6). De même, une observation similaire se retrouve à nouveau plus à l'est avec le sarcophage 3223 qui présente un épandage de mortier très localisé contre la paroi nord de la cuve. Ces dépôts peuvent être interprétés comme de probables témoins des travaux liés à leur installation respective, les cuves étant généralement scellées et jointoyées avec un mortier de chaux mêlant cailloux et fragments de tegulae. Toutefois, aucun niveau de sol construit et extensif n'est attesté dans l'emprise de fouille, notamment aux abords des maçonneries qui adoptent une mise en œuvre semblable. Peu larges (entre $0,55 \mathrm{~m}$ et $0,60 \mathrm{~m}$ ), les arases témoignent de constructions montées en moellons de grès (gris) ou grauwacke (ocre) de modules généralement cubiques et liés au mortier de 


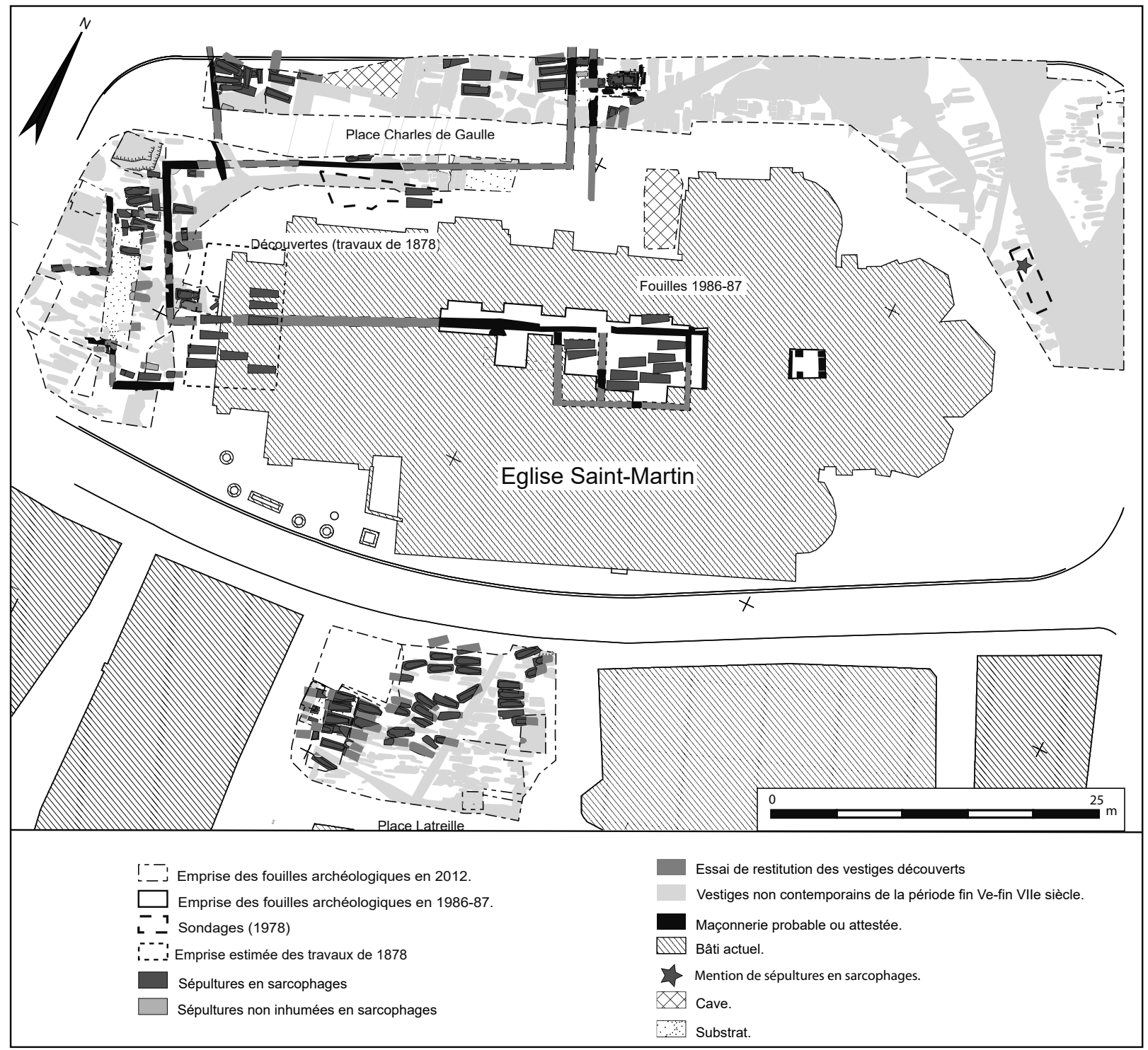

Fig. 4 : Répartition des vestiges découverts (fin Ve-VII e siècle). DAO : E. Barbier, F. Chevreuse, K. Lagorsse, Inrap.

chaux de couleur blanchâtre. Seul le sondage profond réalisé au centre du parvis a permis de préciser au mieux le mode de construction de la maçonnerie M122 fondée sur 3 lits de galets liés à la terre et déposés au sein d'une tranchée étroite. Leur agencement est désordonné et s'adapte au mieux à la diversité des gabarits employés pour le montage des maçonneries. La mise en œuvre évolue pour l'élévation montée avec des moellons en grès local dont le module est généralement cubique, l'ensemble étant lié au mortier de chaux (fig. 7). La limite de cote de fond de fouille n'a pas permis d'évaluer les relations entre les différentes maçonneries à l'exception des murs M135 et M128, parfaitement liés entre eux. Toutefois, le mode de construction et le gabarit similaire des différentes constructions sont autant d'éléments susceptibles d'identifier un programme commun, contrairement aux observations qui avaient pu être menées au sein de la nef actuelle de l'église Saint-Martin

Une sépulture se distingue au sein de cet ensemble funéraire. Située au nord, elle est installée dans le prolongement de l'alignement E/O formé par les autres sarcophages et occupe une position plus ou moins isolée. En effet, une étroite paroi composée de tegulae posées de chant et liées à un mortier d'argile est partiellement conservée sur les pourtours de la cuve en complément d'une maçonnerie (M160) au sud, contre laquelle la cuve s'appose (fig. 5 ; fig. 11). En dépit d'une conservation très lacunaire, l'hypothèse d'une construction maçonnée composée de matériaux en réemploi et délimitant un aménagement de type mausolée peut être envisagée.

Un second groupe d'inhumations en sarcophages fut très partiellement observé au sud de l'église, à l'emplacement du futur enfouissement d'un réseau. Ainsi, l'emprise dégagée n'excédait pas $10 \mathrm{~m}^{2}$, mais elle a toutefois révélé 15 sépultures en sarcophages adoptant la même orientation, globalement est-ouest (fig. 8). La topographie funéraire paraît toutefois complètement différente. Elle se manifeste par une absence de maçonneries, les sépultures étant organisées en rangées très serrées sans possibilité de circulation entre les différentes cuves. Au 


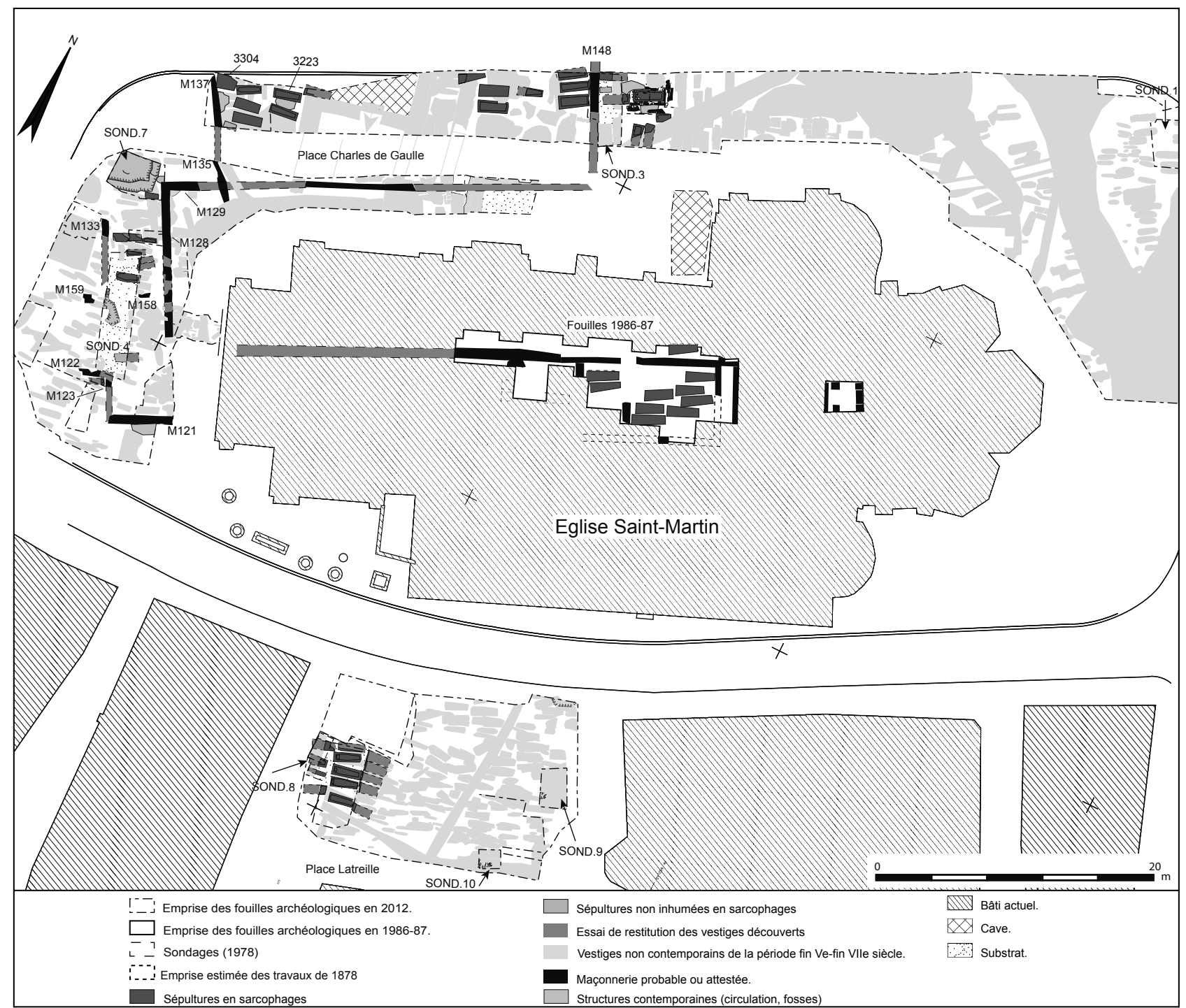

Fig. 5 : Sépultures de la première phase. DAO : E. Barbier, F. Chevreuse, K. Lagorsse, Inrap.

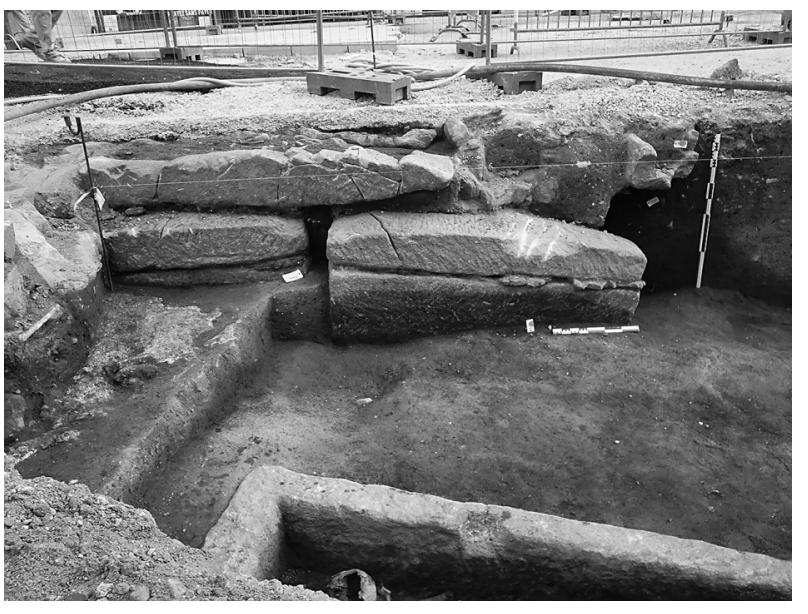

Fig. 6 : Épandage de mortier de chaux observé à proximité du sarcophage 3304. (C) Inrap.

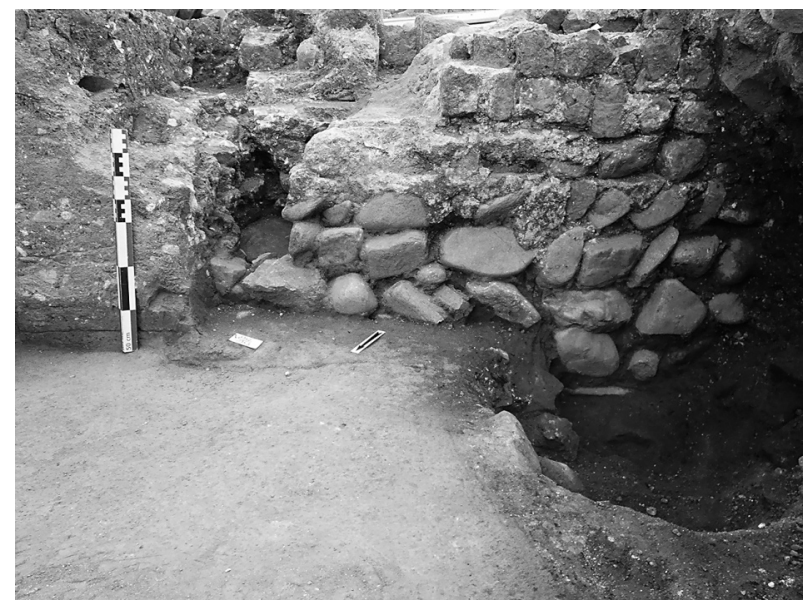

Fig. 7 : Aperçu de la fondation en galets de la maçonnerie M 122. (C) Inrap. 


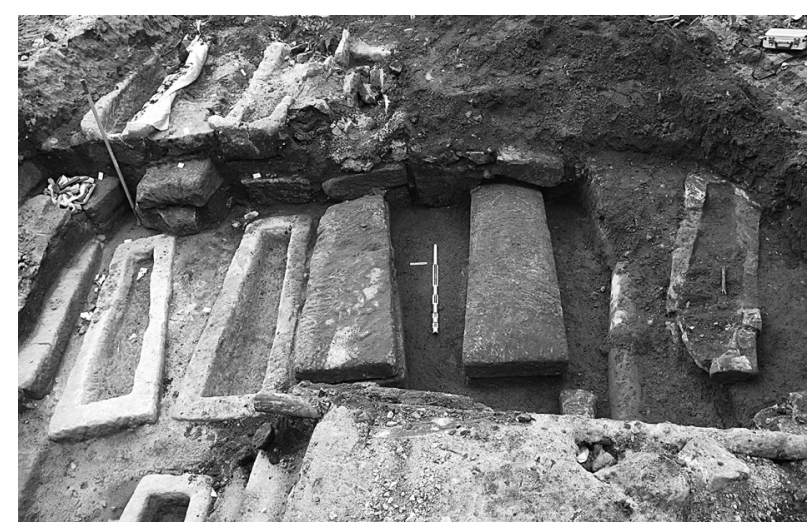

Fig. 8 : Deux niveaux d'inhumations en sarcophages préservés au sud de l'église. (C) Inrap.

moins trois rangées de sarcophages disposés selon une symétrie quasi parfaite peuvent être identifiées. La rangée perçue comme centrale comptabilise au moins 6 sépultures jointives suggérant une très forte densité d'inhumations sur cette partie de la nécropole.

\section{Un aperçu de la population inhumée}

Seuls 21 sujets ont pu être fouillés pour cette première période d'inhumations, donnant une lecture statistique peu représentative de la population inhumée. Toutefois, aucune spatialisation de l'espace ne se distingue d'un point de vue biologique. Sujets adultes et immatures se côtoient et, parmi les adultes, hommes et femmes sont présents ainsi que des adultes jeunes, matures et âgés. Cependant, le sexe n'a pas pu être déterminé pour de nombreux adultes (11/18), et 12 sur 18 n'ont pas de classe d'âge précise.

Parmi ces individus, le soin manifesté pour l'inhumation d'un sujet adulte de sexe masculin (3223) mérite d'être évoqué. Sujet à de nombreuses pathologies, une adaptation atypique du contenant funéraire a été privilégiée. Outre l'ankylose du poignet (7), le défunt présentait au niveau du membre inférieur droit un traumatisme ayant entraîné la fracture des os de la jambe et une dislocation de l'articulation du genou (fig. 9). Celui-ci a imposé une rigidité du genou replié en permanence. $\mathrm{Ne}$ souhaitant pas contraindre cette position et, le cas échéant, briser la jambe afin de l'insérer dans le sarcophage, le fossoyeur a préféré recreuser le fond de la cuve adapté à la taille du pied. Ce geste funéraire atypique, s’il ne peut pas être réellement interprété, implique un respect du défunt qui pose, de fait, la question de son statut. A l'exception de cet individu, les autres sépultures ne témoignent pas d'aménagement particulier. Les défunts sont inhumés en décubitus dorsal, un contenant textile souple étant par ailleurs soupçonné pour 4 individus, dont 2 sujets immatures. Aucun mobilier n'était associé aux défunts.

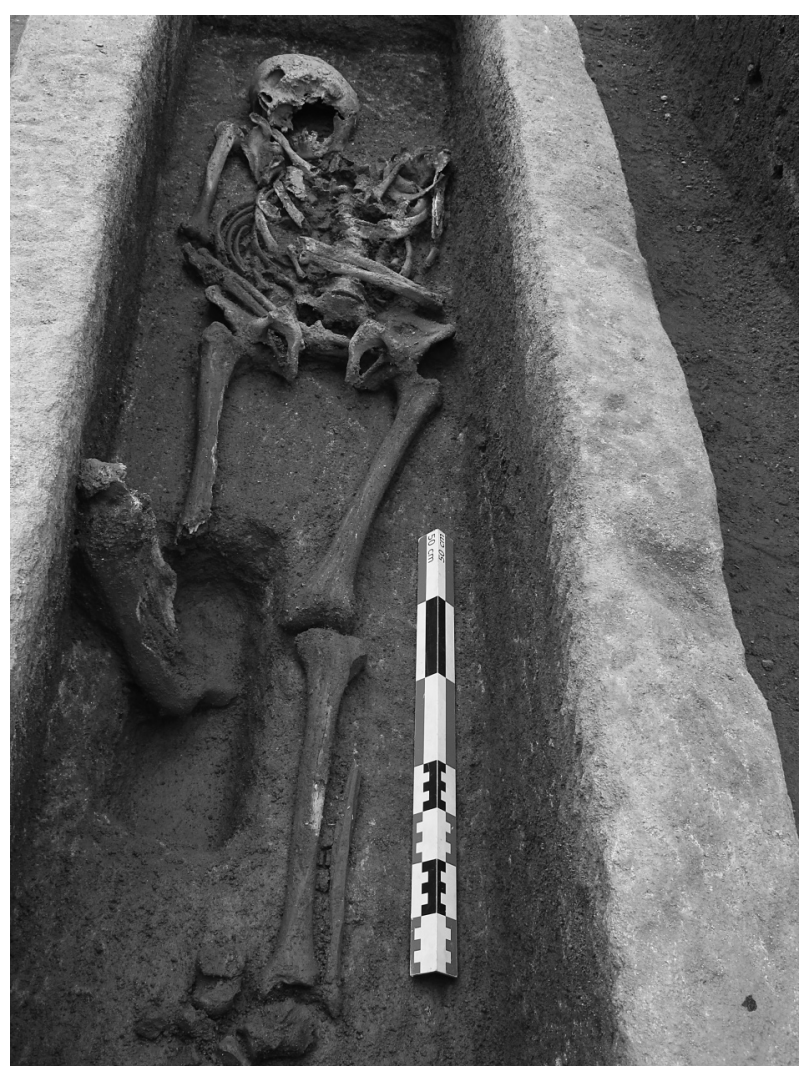

Fig. 9 : Individu infirme inhumé au sein d'un sarcophage adapté à l'une des pathologies du défunt. C Inrap.

\section{Aperçu du premier niveau de sarcophages}

Le premier niveau d'inhumations en sarcophages (le plus ancien) n'a pu être observé que par l'intermédiaire de sondages (8), ce qui limite fortement les conclusions à son sujet. Seule la zone au nord de l'édifice, correspondant à la zone du cloître, a permis d'effectuer des observations sur une zone plus importante. Les données sont très incomplètes bien que des remarques concordantes soient possibles entre ces différents sondages. Pour autant, si l'on ignore la représentativité de cet échantillon, de nombreuses caractéristiques similaires sont présentes et confère à l'ensemble une homogénéité sur la morphologie au premier abord. Les variations observées sont faibles et tiennent souvent du détail : on peut ainsi établir l'existence de trois groupes, désignés $\mathrm{A}, \mathrm{B}$ et $\mathrm{C}$ et détaillés ci-dessous.

Les deux premiers groupes sont très proches et très peu trapézoïdaux, leurs dimensions sont plus importantes que les autres sarcophages du site. Le groupe A est représenté par 6 sarcophages, situés dans le secteur nord de la collégiale. Une bâtière marquée à quatre pans caractérise les couvercles de ces contenants (fig.10), les cuves étant sub-rectangulaires (l'écart maximum entre la paroi de tête et celle de pied est de 11 centimètres). De dimensions importantes (217 à 226 centimètres de

\footnotetext{
${ }^{7}$ Les causes de cette ankylose peuvent être diverses : réaction infectieuse, bactérie pyogène...etc.

${ }^{8}$ Sondage 4 pour le parvis et sondage 8 pour la place Latreille.
} 


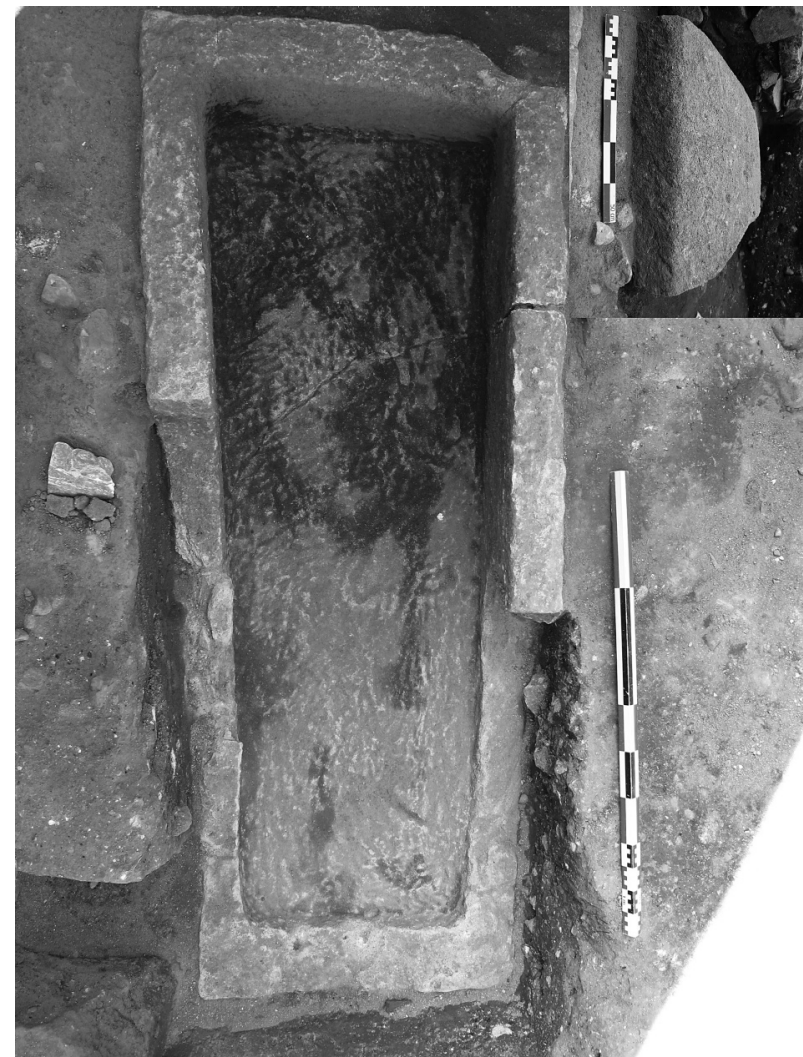

Fig. 10 : Cuve sub-rectangulaire du sarcophage 3280 et détail du couvercle (C G. Rougé).

longueur), ces cuves massives ont des parois épaisses (entre 12 et 15 centimètres pour la majorité). Il s'agit ici probablement des contenants les plus anciens du site ( $I V^{e}$-fin du premier tiers du $V^{e}$ siècle). Le groupe $B$ ne comporte qu'un seul sarcophage (3120), similaire aux exemplaires du groupe A. Seul le caractère bipartite de la cuve le distingue des autres contenants (9). Même si la forme du couvercle est inconnue, il devait se rapprocher de celui des sarcophages du groupe A. Ce contenant possède en outre un aménagement singulier : il est entouré d'une fosse comblée de pierres, tuiles et mortier qui lui est liée : sa fonction reste cependant indéterminée (fig. 11). La forme quasi rectangulaire des sarcophages est principalement marquée au nord et à l'ouest de la nécropole. La zone correspondant à celle occupée plus tardivement par le cloître possède presque exclusivement des sarcophages des groupes A et B. Cette observation mérite toutefois d'être nuancée en raison de nombreuses perturbations postérieures. Les différents sarcophages au sud, dont l'installation est certainement la mieux observée, appartiennent majoritairement au groupe C. Celui-ci, bien que davantage trapézoïdal, est très proche des sarcophages des groupes A et B.

Le groupe $\mathrm{C}$ est l'ensemble le plus important des sarcophages (16 exemplaires) du premier niveau d'inhumations. Présent dans tous les secteurs où des sarco-

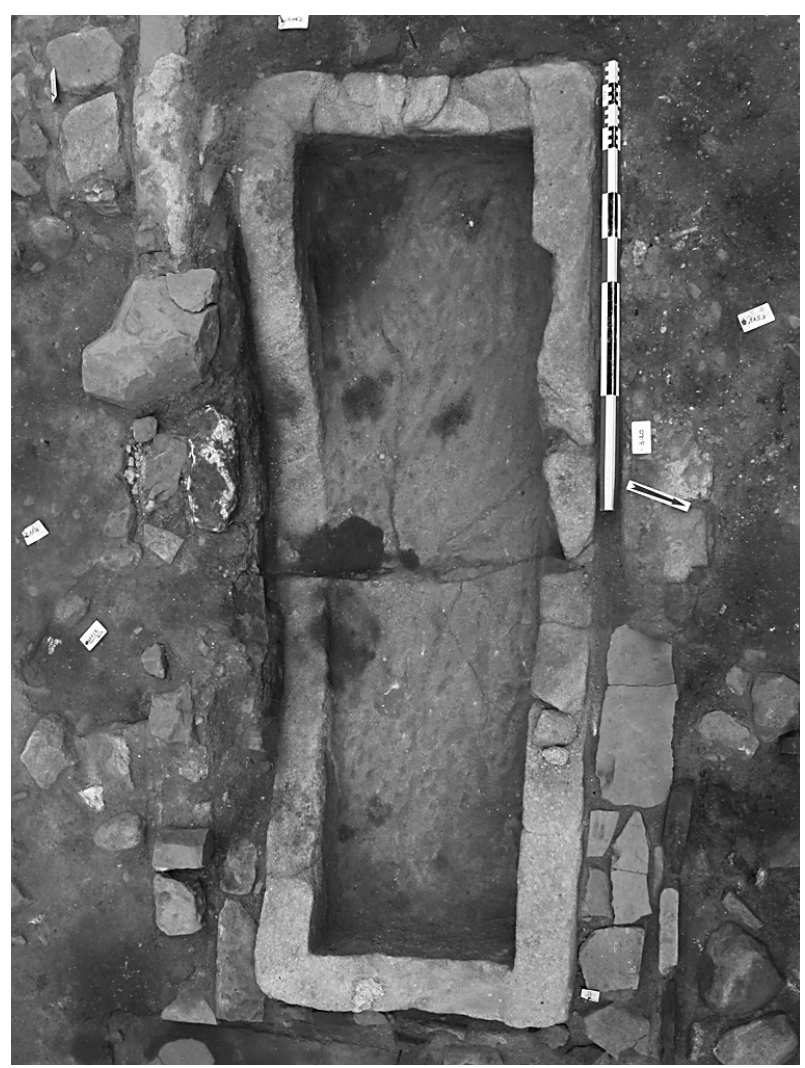

Fig. 11 : Sarcophage bipartite 3120 et son aménagement environnant (C G. Rougé).

phages ont été repérés, les couvercles sont généralement conservés quoique retravaillés pour certains, notamment pour l'installation du second niveau de sarcophages (deux exemplaires en sont témoins : 3226 (fig. 12) et 3625 ainsi qu'une retaille partielle pour le contenant 3305 ) pour l'installation du second niveau de sarcophages. Leur forme première est celle d'une bâtière à quatre pans mais moins prononcée que le groupe A. L'un d'entre eux (3292) possède un " décor » très faible qui consiste à mettre en relief les angles de la bâtière, ainsi qu'un évidement partiel et la présence d'une feuillure chanfreinée permettant l'encastrement sur la cuve (fig. 13). Deux contenants sont scellés au mortier. Une attention particulière est donc décelable pour ce groupe de sarcophages, bien que l'absence de la quasi-totalité des couvercles des groupes A et B ne permettent pas de généraliser cette pratique pour le niveau le plus ancien de sarcophages. Les cuves sont plus petites (largeur et longueur) et la trapézoïdalité des contenants est bien plus importante que les deux groupes précédents. Les différentes études $\mathrm{C} 14$ effectuées situent ces contenants entre le premier tiers du $\mathrm{V}^{\mathrm{e}}$ siècle et le $\mathrm{VI}^{\mathrm{e}}$ siècle.

Ces premières inhumations s'inscrivent au sein d'un espace relativement étendue dont la perception demeure marginale dans le cadre de la fouille archéologique. La population observée ne représente en effet qu'un très

${ }^{9}$ Ce format est présent également dans la Creuse (Delhoume, Roger 2013). 


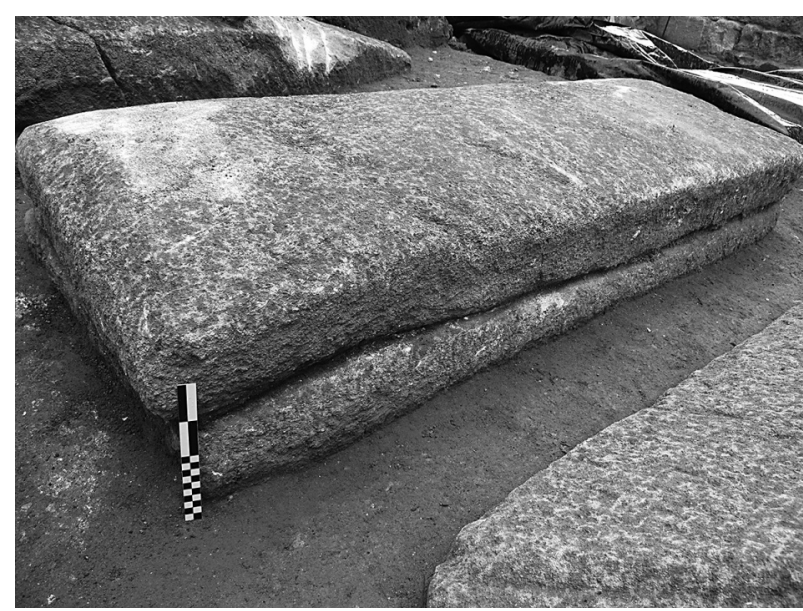

Fig. 12 : Le couvercle du sarcophage 3226 a été retaillé afin de l'aplanir pour permettre l'installation du second niveau de sarcophages (C G. Rougé).

faible échantillon. Cette nécropole s'installe et se développe probablement autour du tombeau de saint Martin dès le $\mathrm{V}^{\mathrm{e}}$ siècle conformément aux données livrées par les 7 datations réalisées sur les ossements pour cette première période (10) et elle se poursuit jusqu'au début $\mathrm{du} \mathrm{VI}^{\mathrm{e}}$ siècle. Tout type de population y est inhumé, sans distinction apparente, mais la topographie funéraire n'est vraisemblablement pas la même sur l'ensemble du site.

A la nécropole ouverte au sud, se confronte une nécropole régie par un ensemble de maçonneries auxquelles s'ajoutent d'autres contraintes moins monumentales. Le bâti semble plus dense au nord de la collégiale et aurait pu délimiter des ensembles clos (type mausolée), mais les informations demeurent trop ténues pour étayer plus précisément cette hypothèse. Au contraire, les sépultures observées au sud de la collégiale relèvent a priori d'une organisation plus extensive.

Des niveaux de piétinements ou de travaux en relation plus probable avec l'installation des cuves sont perceptibles. Ils semblent se différencier des ensembles découverts au sein de la nef de la collégiale, même si l'architecture générale de la première phase d'aménagement identifiée comme telle au sein de la collégiale (Cantié 1987, 71-73) demeure peu documentée. Ils témoignent toutefois d'aménagements internes et probablement clos dont la trame (orientations, modes de construction, gabarits des maçonneries) paraît parfaitement cohérente avec celle révélée par la fouille. Un programme commun de construction paraît donc très probable. Son association avec le développement du culte ad sanctos de saint Martin peut être envisagée. Il aurait précédé l'épiscopat de Rurice I Ir (485-507), contrairement à ce qui était admis.

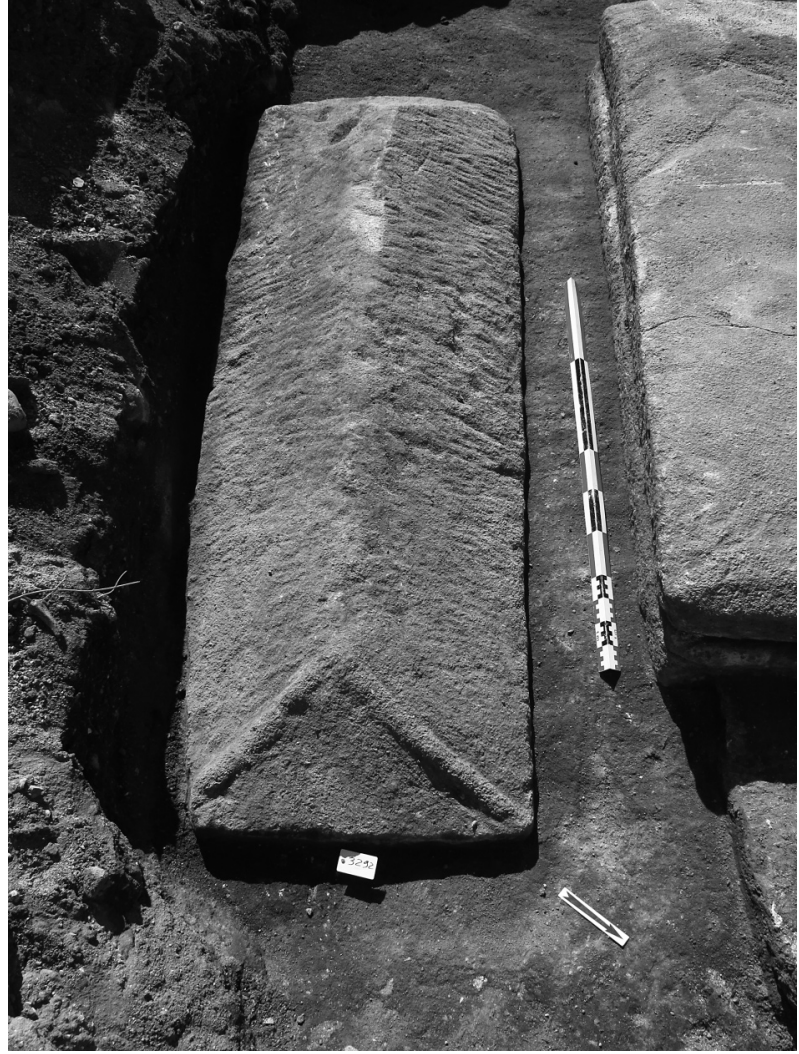

Fig. 13 : Parement du couvercle du sarcophage 3292 et ses arêtes en relief (C G. Rougé).

Le cadre de l'opération archéologique ne permet pas d'appréhender le plan général de ce dispositif qui abrite en son centre des espaces clos et couverts au contraire des espaces périphériques délimités par des maçonneries mais dépourvus de sols aménagés. Ainsi, l'édifice funéraire développé à Brive paraît plus complexe que ce qui avait pu être envisagé à l'issue des fouilles réalisée au sein de la collégiale.

\section{La seconde période d'inhumations (fin $\mathrm{VI}^{\mathrm{e}}$-début $\mathrm{VII}^{\mathrm{e}}$ siècle)}

\section{La topographie funéraire de la seconde période d'inhumations}

Ces espaces funéraires ont été rapidement recouverts par un apport sédimentaire particulièrement dense. Ce phénomène est perceptible depuis le sud jusqu'au nord de l'actuel parvis. Ce dépôt présente de nombreux rejets architecturaux micro-fragmentés (résidus de mortier de chaux, tegulae) plus denses à l'ouest de l'emprise. Ils témoignent de la réhabilitation limitrophe d'un espace non observé dans le cadre de l'opération. Au contraire, la partie sud semble épargnée par ces rejets qui se composent d'une sédimentation limono-sableuse très homogène (fig. 14).

\footnotetext{
${ }^{10}$ Datations Beta Analytic Inc. (intervalles de probabilités à 2 sigmas) : (Âge calendaire). Ouest (parvis) : SP $3310: 426-618$ ap. J.-C. (95,4 \% de proba bilité) ; SP 3143 : 425-611 ap. J.-C. (95,4\% de probabilité). NO (parvis) : SP $3292: 426-618$ ap. J.-C. (95,4\% de probabilité) ; SP $3228: 247-$ 425 ap. J.-C. (95,4 \% de probabilité). Nord église : SP 3127 : 314-433 ap. J.-C. (78,1\% de probabilité). Sud de l'église (place Latreille) : SP 3597 : 326-437 ap. J.-C. (78 \% de probabilité) ; SP 3626 : $425-611$ ap. J.-C. (95,4\% de probabilité).
} 


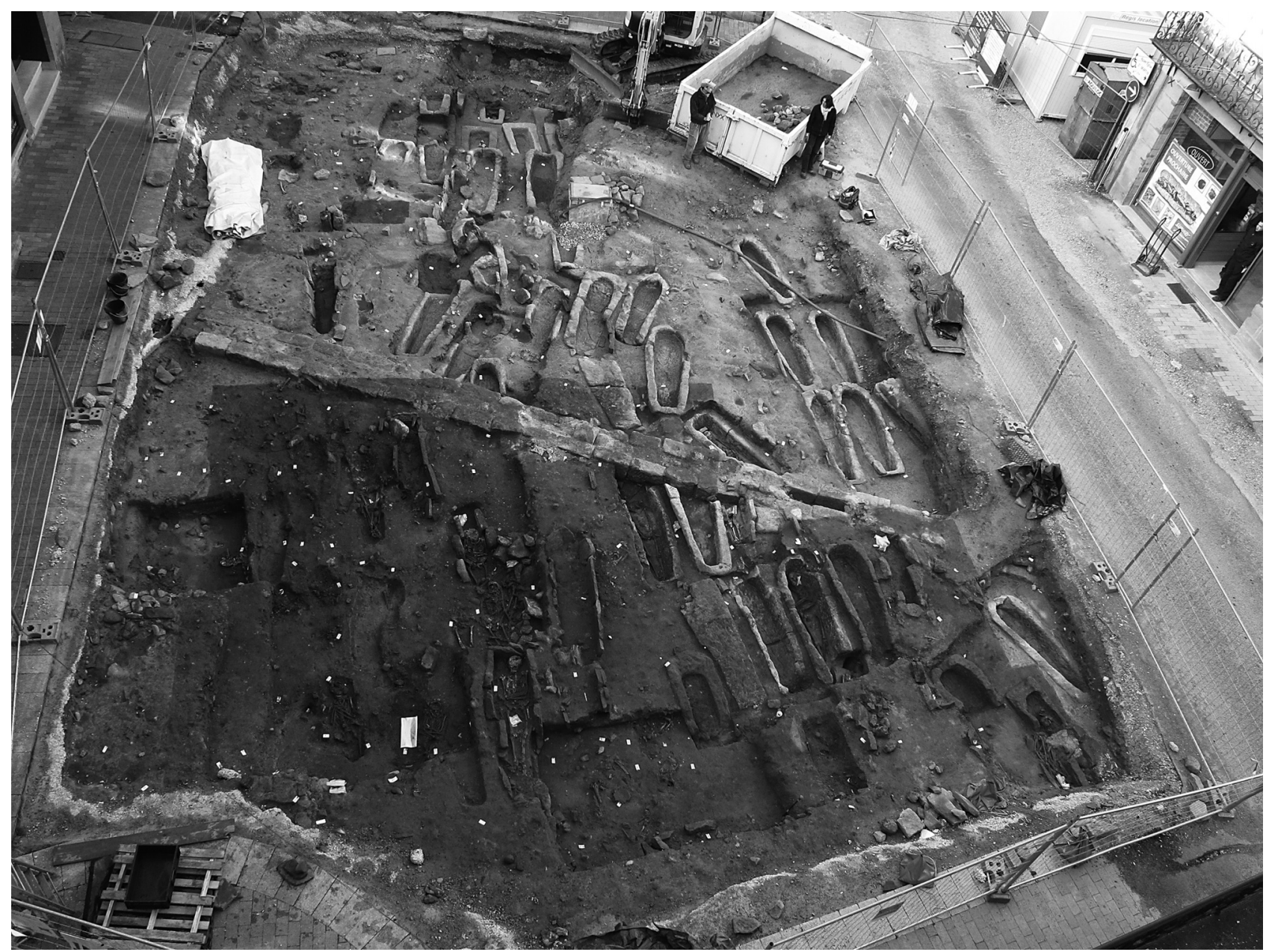

Fig. 14 : Vue générale de la nécropole (sud de l'église). (C) Inrap.

Seul l'espace situé au nord de l'église actuelle semble préservé participant ainsi à la création d'un léger dénivelé qui n'excédait pas une cinquantaine de centimètres entre la partie ouest de la nécropole et le secteur situé au nord. Peut-être la présence d'inhumations plus « prestigieuses », telle la sépulture en sarcophage complété d'un possible mausolée, a imposé cet immobilisme du paysage funéraire sur cette partie du site ? En l'état des connaissances, cette hypothèse ne peut pas être davantage développée.

Les ensembles funéraires identifiés au cours de la première phase d'inhumation ne semblent donc pas modifiés, les mêmes contraintes spatiales étant maintenues (fig. 15). Les sépultures sont installées dans ce nouveau dépôt sédimentaire au contact immédiat des couvercles des premières inhumations. 76 sépultures (pour 77 individus) ont été mises au jour pour cette seconde période. 69 étaient inhumées au sein de sarcophages en pierre (11).

En dépit de perturbations dues aux aménagements postérieurs, ceux-ci ne semblent pas avoir atteint les cuves. Ainsi, des variations sont perceptibles dans l'orientation des sépultures du premier ensemble. Cellesci obéissent plutôt à un besoin de s'insérer au mieux au sein d'un espace dédié dont la matérialisation demeure méconnue à l'exception des quelques maçonneries qui participent toujours à la délimitation d'espaces contraints. Ce phénomène est particulièrement présent à l'ouest et au nord-ouest de l'église actuelle où des secteurs funéraires densément occupés côtoient des espaces «vierges » d'occupation. Des niveaux de sols sommairement aménagés sous la forme d'un galetage (us 1049) ont été observés uniquement sur la partie sud du «parvis» au contact d'une maçonnerie contre laquelle sont inhumés trois individus en sarcophages. Cet aménagement atteste d'une circulation établie au même niveau que les couvercles, mais il demeure marginal puisqu'il n'est nullement présent sur le reste de l'emprise fouillée. L'absence de recoupement entre les différentes fosses sépulcrales de la période tend à indiquer que celles-ci étaient toutefois visibles.

Contrairement à ce qui avait pu être observé au cours des fouilles de la nef, l'espace funéraire ne paraît pas modifié au cours de cette période puisqu'on se contente

\footnotetext{
${ }^{11}$ Les architectures funéraires n’ont pas pu être déterminées pour les autres sépultures.
} 


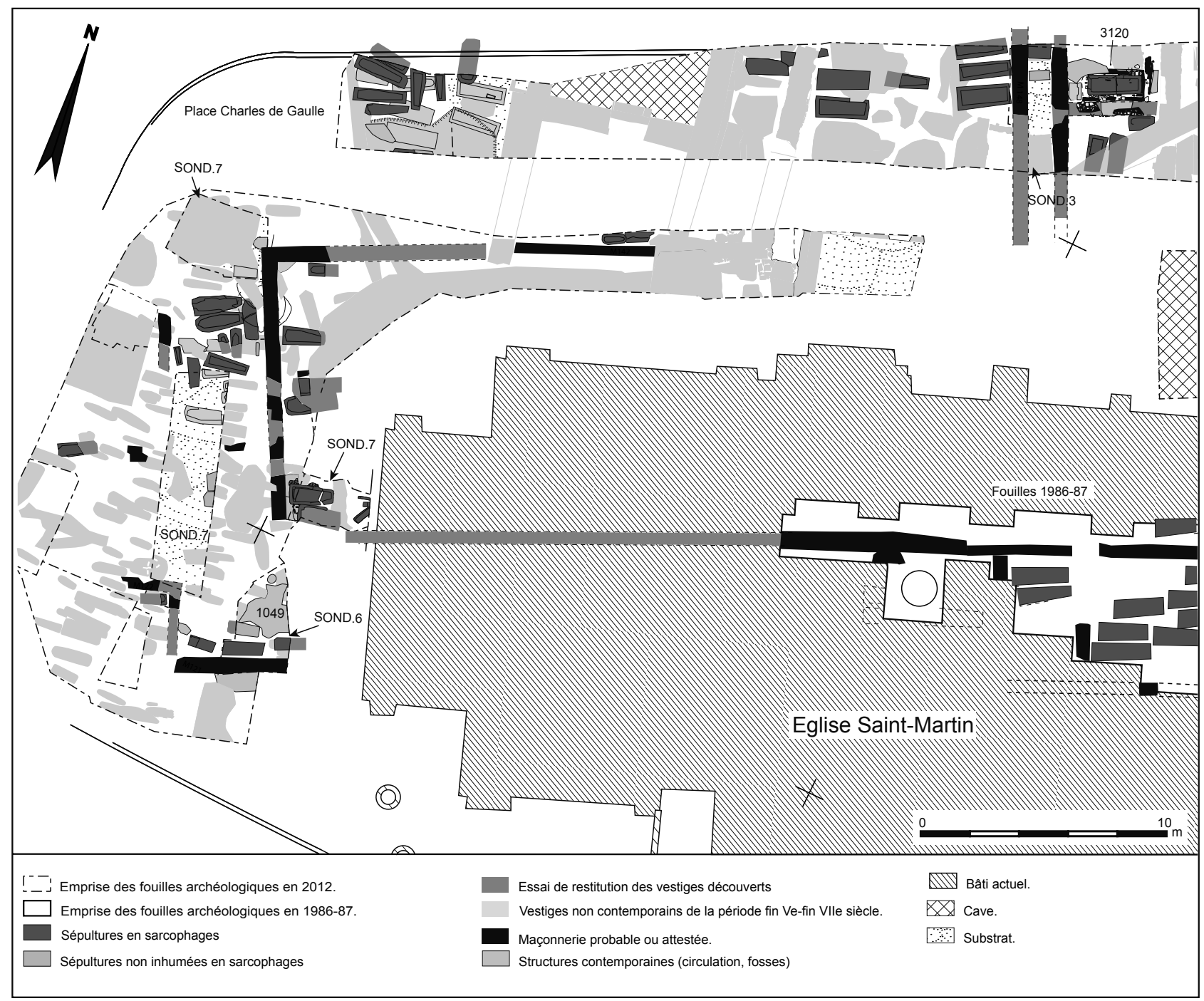

Fig. 15 : Seconde phase d'inhumations en sarcophages aux abords de l'église (VIe-début VII siècle). DAO : E. Barbier, F. Chevreuse, K. Lagorsse, Inrap.

d'inhumer au sein des mêmes lieux, sans reprise du bâti. Seule la maçonnerie M137, qui bordait les sépultures du nord-ouest, semble avoir été abandonnée au cours de cette seconde phase.

La partie sud de la nécropole a pu bénéficier d'une étude plus exhaustive que celle réalisée pour le premier niveau. Près de 43 inhumations, majoritairement en sarcophages (42/43), y sont installées selon des rangées très resserrées limitant d'autant toute circulation (fig. 14, fig. 16). Seule la partie sud de l'emprise ne présente pas d'inhumations en sarcophages suggérant ainsi une limite potentielle au développement de cette aire funéraire qui doit toutefois être nuancée étant donné la limite de la cote de fond de fouille. La réalisation d'un sondage profond à l'ouest de la place montre que le second niveau de sépultures est installé au contact immédiat du premier, les couvercles servant d'assise aux cuves (fig. 9).

L'orientation des sépultures diffère selon les espaces malgré l'absence de contrainte physique avérée (mur, clôture). Ainsi, deux ensembles distincts peuvent être observés. Le premier, consiste dans un agencement réglé des inhumations en rangées régulières. Au moins sept rangées de sarcophages jointifs orientés $\mathrm{E} / \mathrm{O}$, à l'instar de l'église actuelle, ou NO/SE rassemblent près de 32 individus. L'espacement entre chacune des cuves demeure très étroit. La plupart sont jointives et n'autorisent pas de circulation entre les différentes inhumations, comme l'atteste l'absence de niveau de sol sur cette partie de la nécropole.

Cet agencement très réglé se confronte à une disposition plus aléatoire installée selon un axe SO/NE. Il compte 10 sépultures en sarcophages obéissant à des orientations moins rigoureuses que celles précédemment installées. Certaines sont orientées NO/SE au contraire d'autres ( $\mathrm{SO} / \mathrm{NE})$. Ces différentes inhumations semblent donc s'installer au sein d'un espace résultant de contraintes spatiales imposées par des phases distinctes de développement de la nécropole dont la matérialisation n'a pas été appréhendée dans le cadre de l'opération. L'hypothèse d'un cheminement en relation avec l'installation progressive et réglée des différents contenants d'une première période d'inhumation pourrait être envisagée. Ce dernier aurait été progressivement comblé afin 


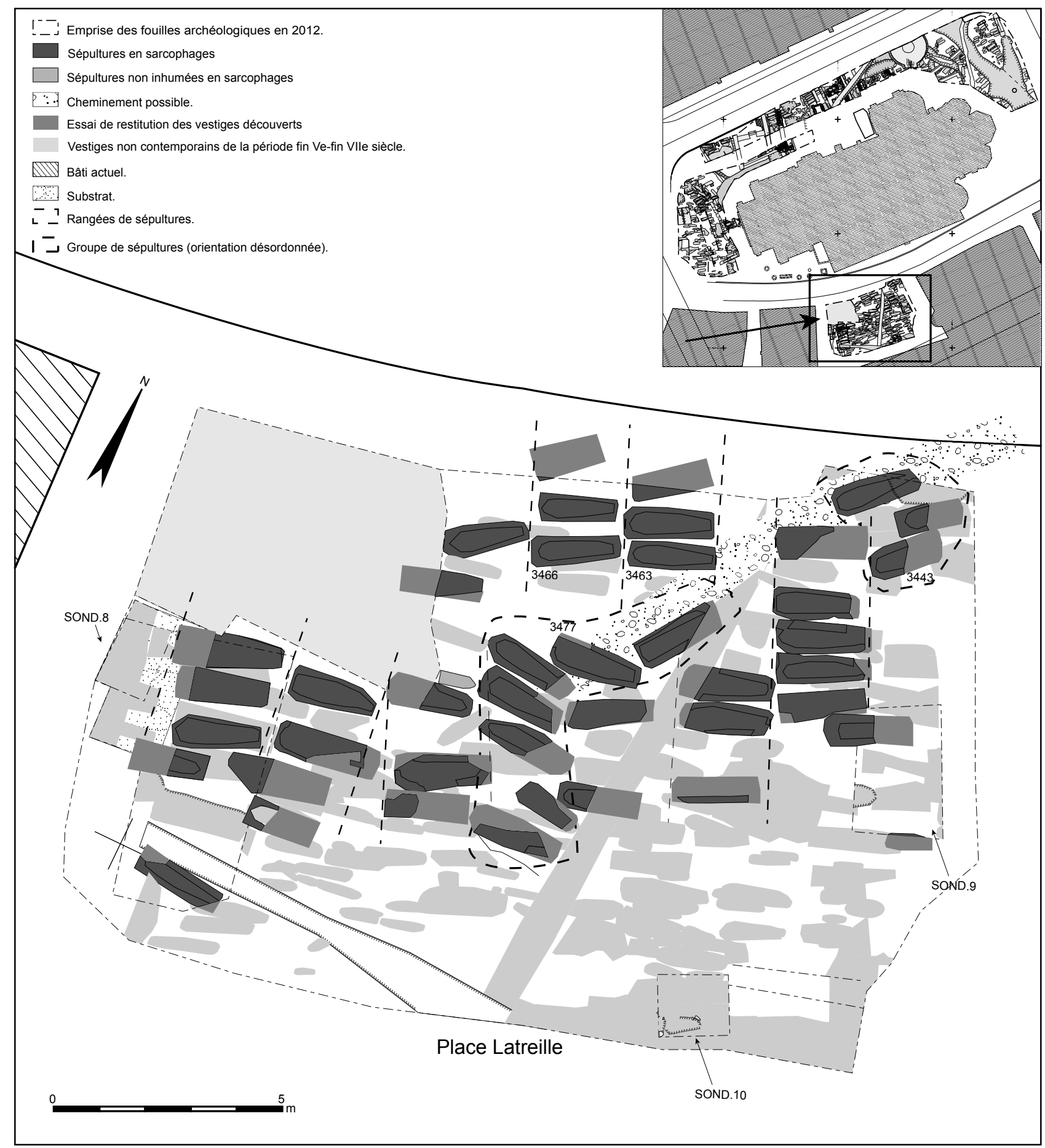

Fig. 16 : Seconde phase d'inhumations (VI'-début VII siècle) au sud de la nécropole. DAO : E. Barbier, F. Chevreuse, K. Lagorsse, Inrap.

de satisfaire aux besoins constants d'inhumer au sein de la nécropole. L'espace demeure ouvert à notre connaissance étant donné l'absence de contrainte (murs et sols).

76 sépultures (pour 77 individus) ont été mises au jour pour la période des $\mathrm{VI}^{\mathrm{e}}-\mathrm{VII}{ }^{\mathrm{e}}$ siècles, correspondant à la seconde période d'inhumation. Comme pour la première, l'architecture funéraire principale demeure le sarcophage, représentant $88 \%$ des inhumations, soit 67 sépultures sur 76. Elle n'a pas pu être déterminée pour six d'entre elles (mauvais état de conservation ou absence de fouille des sujets). Parmi les autres tombes, l'aménagement d'un coffrage mixte pour un individu adulte est reconnu au sein d'un environnement sépulcral pourtant composé exclusivement de sarcophages au nord-ouest. Quant aux deux autres sépultures, elles ont été placées au sein de contenants périssables. Il s'agit pour l'un sujet périnatal et d'un adulte pour l'autre, dont la fosse sépulcrale est perturbée par l'installation d'une autre sépulture en sarcophage. 


\section{Un aperçu de la population inhumée}

La population inhumée, pour la période des $\mathrm{VI}^{\mathrm{e}}$ $\mathrm{VII}^{\mathrm{e}}$ siècles, est assez classique malgré un déficit en immatures (11). L'âge de la plupart des adultes n'a pas pu être appréhendé avec précision. Parmi les 36 adultes observables, 7 sont des hommes, et 7 des femmes, le reste ne pouvant pas être déterminé. Les sujets des deux sexes se répartissent sur l'ensemble des zones étudiées pour la période. Les âges des adultes ne semblent pas non plus être un critère de distinction car jeunes, âgés et matures se côtoient sans partition apparente.

La disposition des individus dans les sépultures est homogène, puisque tous les individus observables sont sur le dos, membres inférieurs en extension.

Quelques caractéristiques au niveau des pratiques funéraires ont été notées, mais celles-ci concernent essentiellement le sud de la nécropole. Tout d'abord, les réutilisations de contenants semblent marginales car seules deux réutilisations sont attestées. De même, seules deux tombes présentent des indices de pillage. La majorité des sépultures relèvent vraisemblablement d'une inhumation primaire et constituent donc un échantillon assez fidèle des pratiques funéraires. Parmi celles-ci, l'utilisation d'un contenant textile souple pour un sujet immature a notamment été observée. La présence d'un brancard ou lit funéraire est également envisagée pour un individu inhumé en sarcophage (3466). Une pratique plus répandue est la surélévation du crâne du défunt, puisque 20 sujets sur 24 observables ont la tête surélevée, la mauvaise conservation, voire la destruction du bloc crânio-facial et/ou des vertèbres cervicales, ayant empêché une observation plus conséquente de cette pratique funéraire. Cette pratique parait toutefois plus répandue dans la partie sud de la nécropole au sud où 17 individus en sarcophages sur 20 observables ont la tête surélevée (pour 44 individus au total).

A l'inverse, le mobilier est quasiment absent au sein des tombes. Seuls trois individus étaient inhumés sur la partie sud de la nécropole avec des éléments de parure (fig. 17). Sans détailler ces vestiges, signalons :

- Le sujet 3463-3 portait un anneau à la main gauche, tandis qu'un petit anneau a été retrouvé sous le thorax (détail vestimentaire ?).

- Le sujet 3443-3 portait également une bague avec un plateau rectangulaire portant des motifs en creux de croix et palmettes (12).

- Le sujet 3477-3 qui portait une bague à la main droite avec un possible bracelet au poignet gauche (13).

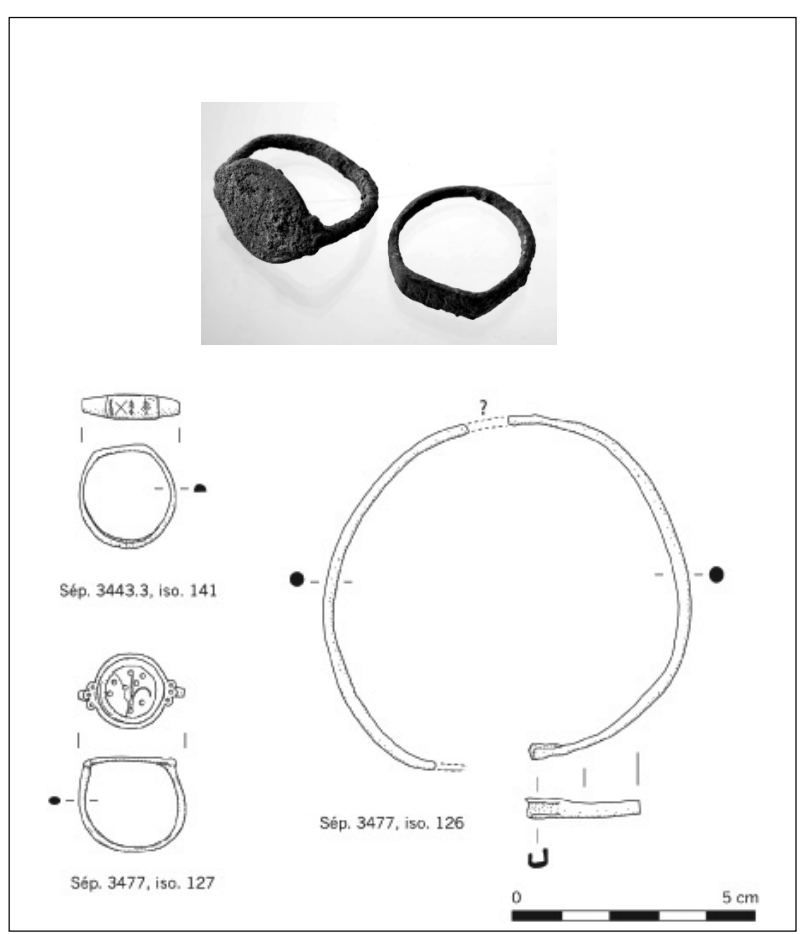

Fig. 17 : Eléments de parure découverts au sein des sépultures de la seconde période d'inhumations (sépultures 3443 et 3477). Cliché : P. Ernaux, Inrap ; Dessins : I. Bertrand, musée de Chauvigny.

En conclusion, la seconde période d'inhumations présente des caractéristiques biologiques et des pratiques funéraires très homogènes. Près de 8 datations $14 \mathrm{C}$ ont été réalisées à partir des ossements des défunts (14) confirmant l'échéancier très court entre ces deux phases distinctes. La seconde semble, en effet, s'être développée dès la seconde moitié du VI ${ }^{\mathrm{e}}$ siècle tout se poursuivant au siècle suivant. Toutefois, aucune inhumation n'est reconnue au-delà de cette période.

\section{Les sarcophages du second niveau : une variabilité plus importante}

Le second niveau d'inhumations est celui qui a livré le plus grand nombre de sarcophages (répartis en quatre groupes) au sein de la nécropole qui s'est développée autour de la collégiale Saint-Martin. A l'unité typologique du premier niveau, celui-ci présente une variété bien plus importante. Le matériau employé reste le même (le brasier, un grès local) et la technique de fabrication ne semble pas différer de la période précédente, en

\footnotetext{
${ }^{12}$ Étude du mobilier réalisée par Isabelle Bertrand, Musée de Chauvigny (86), chercheur associé UMR 5140, Lattes, équipe TP2C ; Équipe HeRMA, EA 3811, université de Poitiers (Barbier et al. 2015, vol. 2, 158). Bague de type Hadjaj 3a : Ve-début VIII ${ }^{\mathrm{e}}$ siècle.

${ }^{13}$ Étude du mobilier réalisée par Isabelle Bertrand, Musée de Chauvigny (86), chercheur associé UMR 5140, Lattes, équipe TP2C ; Équipe HeRMA, EA 3811, université de Poitiers (Barbier et al. 2015, vol. 2, 158). Corps de section circulaire aplati au niveau inférieur ; sa partie supérieure forme un plateau circulaire $(15,8 \times 16 \mathrm{~mm}$; ép. $1,3 \mathrm{~mm})$ dont le pourtour est souligné de deux rainures et au centre duquel figure un motif linéaire alternant avec des motifs circulaires. Les épaules sont soulignées de trois granules portant chacune un point. Découverte près du poignet droit. Type Hadjadj 4a : $\mathrm{VI}^{\mathrm{e}}-\mathrm{VII}{ }^{\mathrm{e}} \mathrm{s}$.

${ }^{14}$ Datations Beta Analytic Inc. (intervalles de probabilités à 2 sigmas) : (Âge calendaire). Ouest (parvis) : SP $3138: 528-642$ ap. J.-C. (75,1 \% de probabilité) ; SP 314 : 548-657 ap. J.-C. (95,4\% de probabilité). NO (parvis) : SP 3225 : 536-658 ap. J.-C. (95,1 \% de probabilité) ; SP 3263 : $440 \pm 40$ ap. J.-C. (âge conventionnel : 95,4 \% de probabilité). ; SP 3227 : 506-636 (69,7\% de probabilité). Sud de l'église (place Latreille) : SP 3507 : $534-654$ ap. J.-C. (95,4 \% de probabilité) ; SP 3538 : 538-658 ap. J.-C. (92,3\% de probabilité) ; SP $3466: 577-690$ (94,2\% de probabilité).
} 
dehors d'une finition moins aboutie sur cette seconde phase. Un petit groupe F se distingue cependant par un matériau différent, en grès rouge. L'évidement des cuves est le plus souvent désaxé et l'épaisseur des parois est variable et non rectiligne : l'intérieur des parois n'est pas parallèle à l'extérieur de celles-ci (fig. 18). La forme globale, trapézoïdale, subit des irrégularités fréquentes (asymétrie quasi systématique), qui affecte principalement le chevet (droit, arrondis ou à pans coupés). Ces derniers points concernent le plus grand nombre des sarcophages du second niveau, à savoir les groupes D et E. Le groupe F est à exclure de ces considérations, l'outil employé et le matériau changent, ce qui induit un travail et une provenance différents. Le groupe G semble bénéficier d'une réalisation bien plus soignée et possède un aménagement céphalique, mais celui-ci n'est représenté que par un seul exemplaire, incomplet de surcroit.

Trente-huit contenants font du groupe D le plus important sur le site et celui qui correspond à un format très fréquent au haut Moyen-Age : trapézoïdal à chevet droit. Comme mentionné plus haut, si l'extérieur ne présente aucune information particulièrement spécifique, il y a davantage à dire sur l'aspect intérieur des cuves. En effet, c'est sur ce groupe que l'on retrouve la plus importante

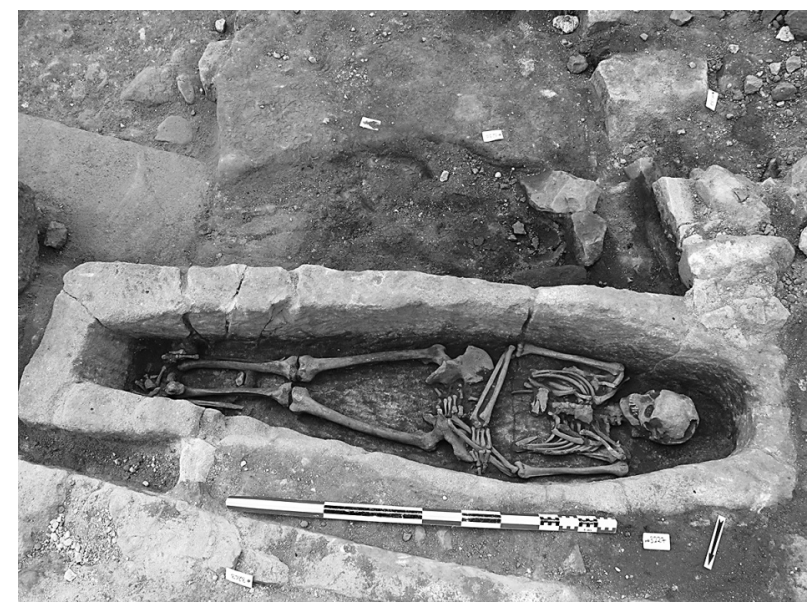

Fig. 18 : Evidement non régulier de la cuve 3227 et installation très exigüe de l'individu (C Inrap).

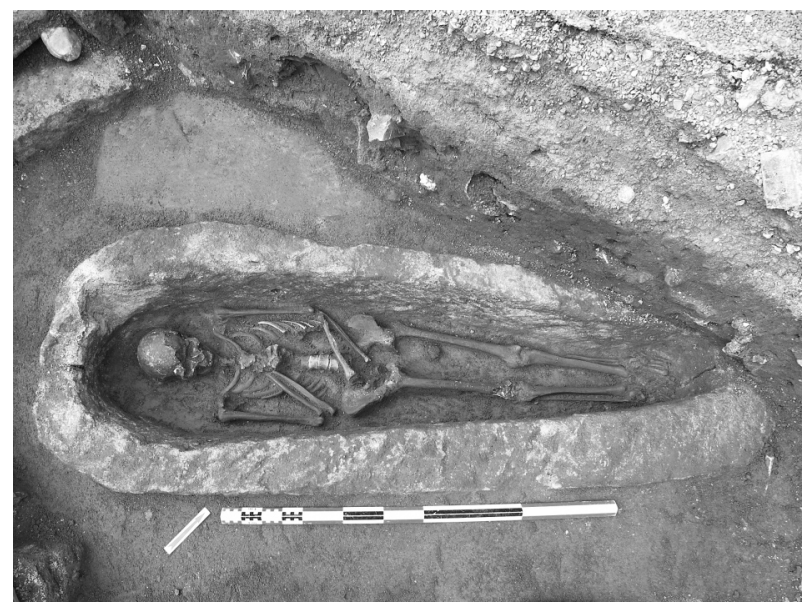

Fig. 19 : Sarcophage à chevet arrondi, n 3452 (C Inrap). part d'évidement désaxé et de parois à épaisseur variable (fig. 18). De plus, ce second niveau a été fortement perturbé et nombreux sont les contenants dont la conservation est très médiocre. Le groupe $\mathrm{E}$ partage un grand nombre de critères avec le précédent : sa différence notable est celle de la forme du chevet des sarcophages : arrondie ou à pans coupés (fig. 19), là aussi avec des dissymétries fréquentes. Il est à remarquer que les couvercles de ces deux groupes, bien que la forme de la cuve les différencie, sont plats, trapézoïdaux, à chevet droit pour tous ceux qui ont pu être observés. Sur l'un d'eux (le sarcophage 3466), une inscription y a été gravée, mais celle-ci est difficilement déchiffrable en raison de la conservation incomplète du bloc et d'une cassure.

Un groupe atypique $\mathrm{F}$ est présent au plus près de la collégiale Saint-Martin de Brive et est constitué de cinq sarcophages : le matériau employé est ici différent de tous les autres contenants. En effet, si le brasier est le matériau " générique », ce groupe est quant à lui réalisé en grès rouge (ou lie-de-vin) (fig. 20). La forme, classique par le plan trapézoïdal à chevet droit, présente un soin plus important dans la finition avec des parois rectilignes, de faible épaisseur et un aménagement céphalique taillé en réserve pour au moins deux cuves. La forme des couvercles est complètement inconnue, aucun couvercle ou fragment n'ayant été retrouvé. Le groupe $\mathrm{G}$ ne concerne qu'un seul sarcophage (fig. 21). Comme pour le groupe $\mathrm{F}$, un aménagement céphalique similaire est présent et ce, à l'intérieur d'un chevet à pans coupés. Sa conservation partielle ne permet pas de connaître la forme générale de la cuve même s'il semble que celle-ci

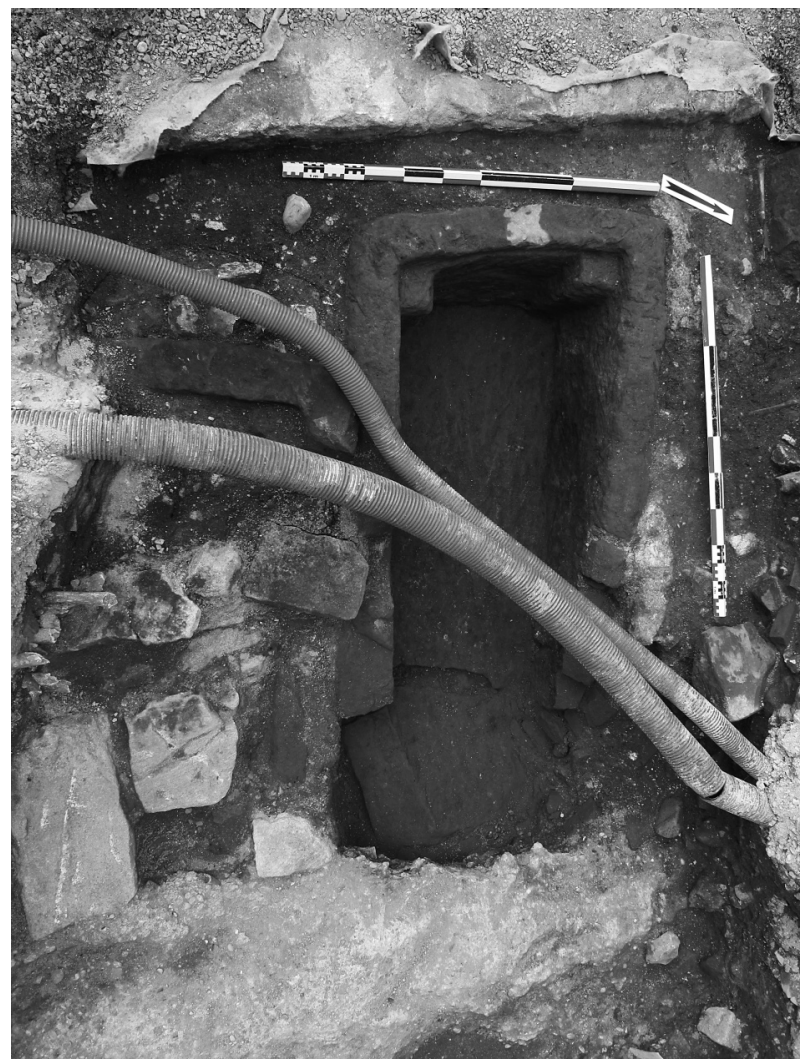

Fig. 20 : Sarcophage en grès rouge $\mathrm{n}^{\circ} 3337$ (C) Inrap). 


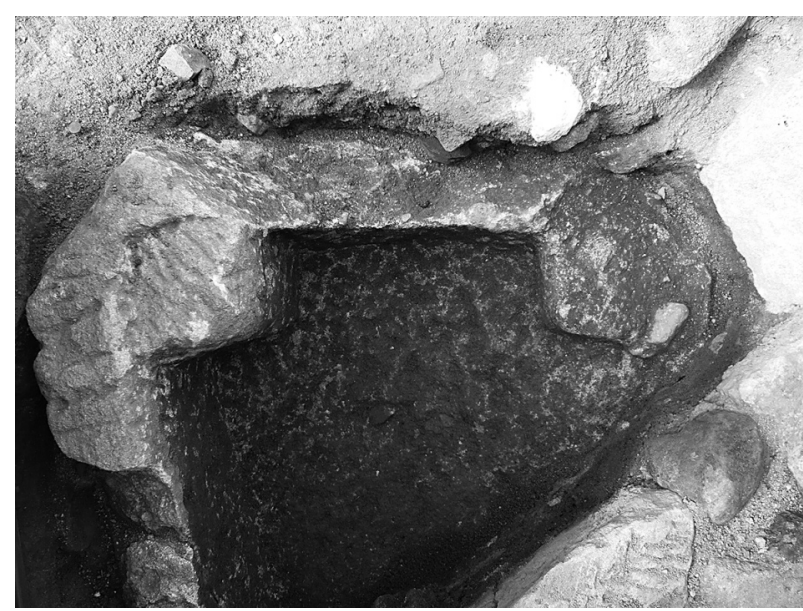

Fig. 21 : Sarcophage $n^{\circ} 3295$ à aménagement céphalique et chevet à pans coupés (C G. Rougé).

soit trapézoïdale. L'utilisation du brasier pour ce groupe montre une production variée du matériau sur le site. La datation, supposée plus tardive au départ, correspond en réalité à la même période que le second niveau de sarcophages : des cuves similaires ont été découvertes également à Tulle (Montigny, 2007) présentant des caractéristiques similaires, et des datations concordantes.

L'organisation globale des sarcophages du second niveau s'inscrit dans la continuité du premier niveau. Quelques exceptions existent au sud de l'espace funéraire ou sur le parvis, sans altérer l'homogénéité de la nécropole. Les groupes $\mathrm{D}$ et $\mathrm{E}$, majoritaires sur ce niveau, sont présents autour de la collégiale, cette observation demeurant toutefois partielle pour le secteur Nord (cloître). La présence exclusive au nord de l'édifice et sur le parvis de sarcophages en grès rouge $(F)$ démontre une utilisation préférentielle de ce matériau au sein de zones dédiées et situées au plus près de l'édifice actuel. Le choix d'un matériau exogène au bassin de Brive et le soin manifesté dans la mise en œuvre des contenants démontrent un usage privilégié qui ne peut toutefois pas être davantage développé étant donné l'état lacunaire et fragmentaire de l'information (15). Les différentes datations proposées pour les différents groupes qui y sont présents donnent une fourchette du début du $\mathrm{VI}^{\mathrm{e}}$ siècle à la fin du VII ${ }^{\mathrm{e}}$ siècle.

\section{Des aménagements spécifiques}

L'ensemble des sarcophages du site de la collégiale ne présente pas d'aménagements habituellement rencontrés, comme des coussins céphaliques, logettes, chanfreins sur des parois, feuillures ou encore des perforations de fond de cuve. Si la conservation des contenants a pu être un obstacle à ces observations, il n'en reste pas moins que le nombre de cuves qui en sont pourvues est presque anecdotique sur le site.
Un exemplaire unique (sarcophage 3292) présente sur le contre-parement du couvercle une association de deux aménagements que l'on ne retrouve pas ailleurs : une feuillure chanfreinée (fig. 22). Il s'agit ici également du seul couvercle avec les angles de la bâtière en relief (est-ce un effet d'ornementation ou le caractère inachevé du couvercle ?). Aucune cuve fouillée n'est perforée en fond de cuve, et les coussins céphaliques sont absents (à l'exception éventuelle du sarcophage 3466). Les logettes céphaliques présentes sur les groupes $F$ et $\mathrm{G}$ consistent en des piles d'angles de dimensions similaires (fig. 23). Quelques autres cuves présentent des aménagements à la tête du type encoche, mais cela reste rare.

C'est un tout autre type d'aménagement qui est majoritaire autour de l'édifice dédié à Saint-Martin. Il s'agit ici d'amaigrissements de parois, qui modulent l'espace intérieur de manière plus ou moins importante, selon

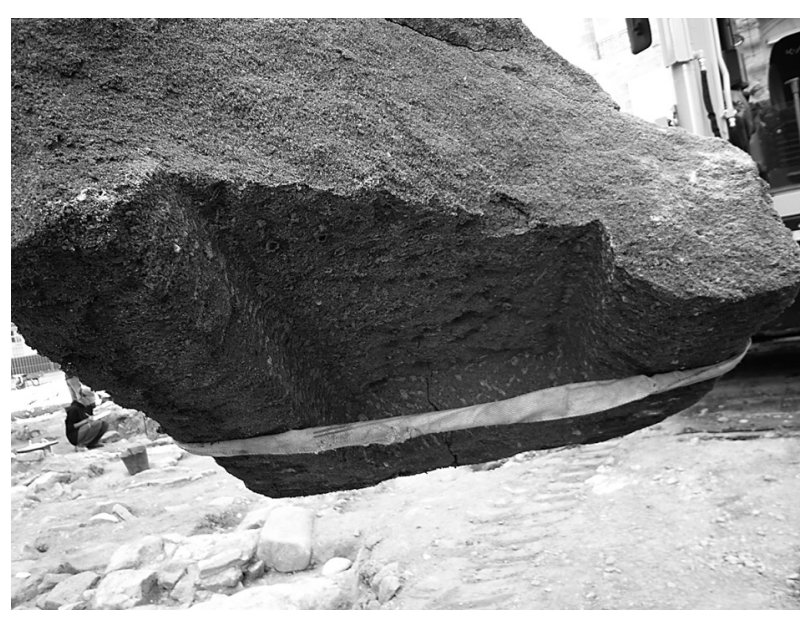

Fig. 22: Feuillure chanfreinée du sarcophage 3292 (C) G. Rougé).

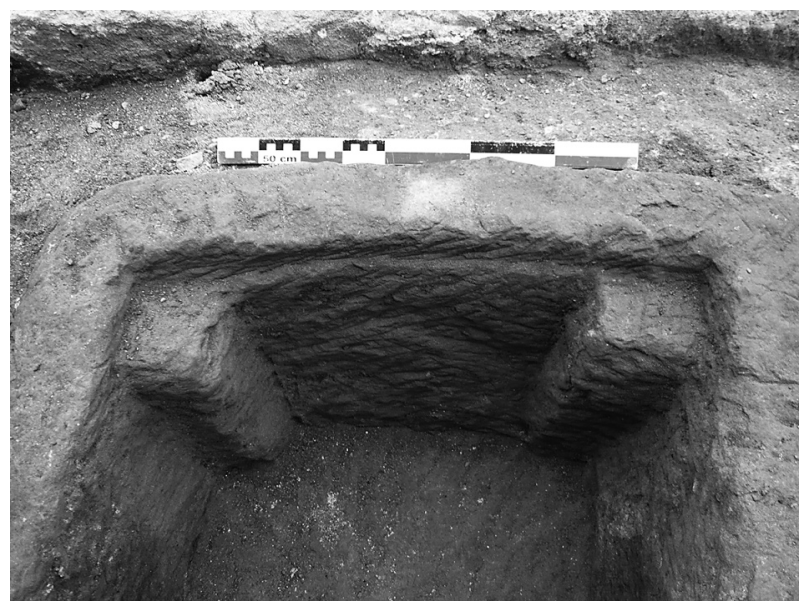

Fig. 23 : Type d'aménagement céphalique avec des piles dans les angles (C) G. Rougé).

\footnotetext{
${ }^{15}$ Le contexte lié à la topographie funéraire de ces sépultures situées en limite d'emprise de fouille et soumises à de nombreuses perturbations récentes n'a pas pu être précisé dans le cadre de l'opération archéologique.
} 
l'épaisseur amoindrie. En effet, ces retailles varient en longueur en profondeur et en positionnement. Ils affectent le plus souvent les parois latérales mais également parfois la paroi de tête. Dans un seul cas, c'est même ce dernier qui est le seul aménagé. Pour le groupe $\mathrm{C}$, neuf cuves sur quatorze sont aménagées de cette manière, l'espace interne y est ainsi élargi afin d'y déposer les défunts. Ces retailles combinées sur les parois et sur celle de tête, visibles sur certains sarcophages, ont presque entièrement effacé les angles d'origine de l'évidement primaire. Ceux-ci ne sont désormais visibles que sur la partie supérieure des parois et leur base est arrondie pour former un contour semi-circulaire à la tête. Cette constatation est fréquente pour le groupe $\mathrm{C}$ mais pas uniquement : 22 cuves, appartenant à différents groupes typologiques (A, C, D et E), sur les 112 sarcophages repérés sur le site, ont été retaillées. Le ratio est déjà important, mais il pourrait être augmenté, car les 112 contenants n'ont pas tous été fouillés et certains n'apparaissaient que très partiellement, en limite d'emprise.

Ces retouches, non systématiques, sont pourtant indispensables pour certaines, l'individu inhumé n'aurait eu la place d'être positionné sur le dos. La zone entre les épaules et le bassin du défunt est celle qui est retravaillée le plus souvent, mais on constate également des retouches plus importantes entre les genoux et les épaules, voire jusqu'à la tête, et même la paroi de tête. Au final, certaines cuves ne laissent que peu de traces des angles supérieurs de l'évidement primaire du bloc. Pour un cas, la retaille forme un arrondi complet à la tête, qui ne correspond plus à la forme externe du contenant (fig. 24).

La présence de ces aménagements de parois sur un sarcophage du groupe A (retaille légère sur les parois latérales), puis une récurrence sur le groupe $\mathrm{C}$ et également le groupe $\mathrm{D}$, laisse imaginer une continuité de cette pratique pendant quelques siècles (approximativement entre le $\mathrm{IV}^{\mathrm{e}}$ et le $\mathrm{VII}^{\mathrm{e}}$ siècle). Cet acte intentionnel ne semble pas effectué lorsque le sarcophage est en place dans le cimetière, en raison de l'absence totale de fragments ou déchets de taille dans les cuves. Cependant, un acte réalisé en carrière aurait pu conduire une nouvelle phase de travail qui aurait affiné les cuves en totalité. La fabrication des contenants est, elle aussi, en discussion. Certaines cuves produites sans retaille n'étaient pas fonctionnelles en raison de l'exigüité de l'espace interne, l'élargissement était-il prévu dès l'origine ? Ou bien estce qu'il faut y voir un choix délibéré de produire des cuves avec des parois aussi épaisses ? Fournir des éléments de réponse n'est pas aisé, mais quelques éléments sont à développer. L'épaisseur du fond des cuves est également un point à aborder : lors de la fouille, la possibilité d'extraire des cuves a été liée uniquement à la conservation de celles-ci. Sur celles qui ont pu en bénéficier (groupe $\mathrm{A}$ et $\mathrm{C}$ principalement), un point commun est apparu : l'épaisseur du fond des cuves est importante. Sur les sarcophages calcaires présents à l'ouest de la Corrèze (Rougé 2014), l'épaisseur moyenne se situe entre 10 et 15 centimètres. Pour les sarcophages de Brive, on atteint les 20/25 centimètres de manière fréquente, si ce n'est davantage (16). Si l'on considère que les parois sont plus épaisses pour faciliter le transport des sarcophages, il est alors envisageable d'effectuer une nouvelle phase de travail à proximité du lieu d'inhumation afin d'ajuster l'intérieur des contenants avant leur mise en terre.

Cette question du travail de retaille sur les sarcophages de Brive n'est pas sans intérêt pour la question de la provenance des matériaux. A l'exception du groupe F, réalisé avec un grès rouge que l'on retrouve au nord de Brive, les autres groupes de sarcophages sont produits en brasier (grès arkosique), matériau fréquent à proximité de la commune. Si l'on peut s'interroger sur le fait d'aller chercher plus au nord des sarcophages en grès rouge, on comprend facilement, pour le transport, l'utilisation du brasier local pour produire l'essentiel des contenants.

Ces différentes données attestent donc du maintien d'une importante nécropole autour de la basilique hébergeant la dépouille de saint Martin dit l'Espagnol. Grégoire de Tours témoigne de l'existence de cet édifice muni d'un autel et de colonnes en marbre (cf. supra). Incendié en 584, il aurait été reconstruit à la fin du $\mathrm{VI}^{\mathrm{e}}$ siècle par l'évêque de Limoges, Ferréol, afin d'abriter à nouveau le culte du saint : " ....sed ita hae aedes in posterum a Ferreolo episcopo reparata est, tanquam si nihil mali pertulerit... » (Gregorii Turonensis Historiarum Libri X, 1951, p. 332). Geneviève Cantié attribue à cette période de travaux les reprises de maçonneries et autres traces d'incendie observées sur l'édifice dégagé à la croisée de la nef et du transept actuels. Or, cet événement n'est nullement perceptible au sein des espaces funéraires dégagés au cours de la dernière campagne de fouille. A l'inverse, leur organisation respective est étroitement dépendante de la première phase d'aménagement et du bâti qui lui était associé. Certes, le dégagement superficiel des niveaux nous invite à nuancer cette analyse. Ainsi, les relations entre les différentes maçonneries n'ont pas pu être vérifiées. Toutefois, l'observation, même partielle, de leur mise en œuvre respective témoigne d'un programme cohérent, contrairement aux vestiges découverts au sein de la nef.

L'incendie évoqué par Grégoire de Tours ne semble pas avoir modifié la gestion funéraire des abords du sanctuaire. Celui-ci a pu se concentrer au sein de quelques bâtiments et épargner le reste du site. A cet égard, le soin manifesté dans les travaux de réhabilitation (murs enduits, sols en béton de tuileau) semble s'être limité à la partie « centrale » de la basilique. Les niveaux de sols en galets mis au jour à l'ouest rendent compte au contraire d'aménagements plus « frustres ».

\footnotetext{
${ }^{16}$ Un autre fait, mais qui avait permis de se rendre compte de l'épaisseur des fonds de cuve, concerne le sarcophage 3223 (celui de l'individu dont la pathologie empêchait la fermeture du sarcophage, mentionné ci-dessus). Celui-ci a livré un surcreusement oblong d'une trentaine de centimètres de longueur, proche de la partie pied du contenant. La profondeur de ce trou dépasse les 16 centimètres et en aucun cas, le fond de cuve n'est perforé. L'extraction du sarcophage par la suite montrera une épaisseur dépassant les 30 centimètres, ce qui laissait de la marge pour une action de ce type.
} 


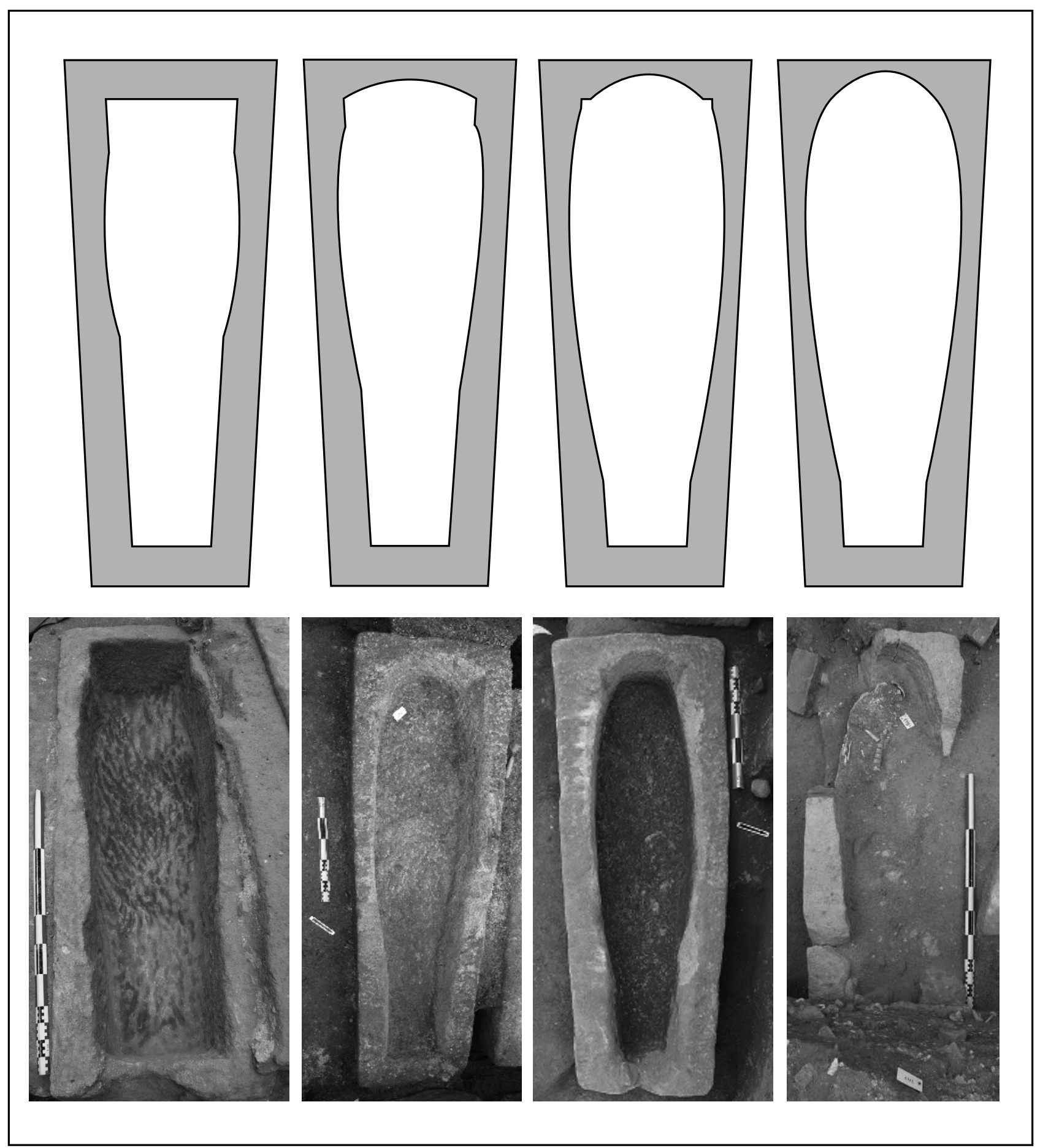

Fig. 24 : Variations des aménagements et élargissements dans les sarcophages (DAO et Cl. G. Rougé).

\section{CONCLUSION}

$\mathrm{Si}$ le cadre de l'opération (côte de fond fouille limitée, contraintes techniques) ne permettait pas une approche exhaustive de l'occupation sur l'ensemble de la période appréhendée $\left(\mathrm{V}^{\mathrm{e}}-\mathrm{VII}{ }^{\mathrm{e}}\right.$ siècle), la qualité de conservation de la plupart des vestiges archéologiques épargnés par les travaux du XIX ${ }^{\mathrm{e}}$ siècle offre de précieuses informations inédites sur les conditions d'implantation et de développement des espaces funéraires depuis la fin du $\mathrm{V}^{\mathrm{e}}$ siècle.
La topographie funéraire est toutefois apparue plus complexe que celle envisagée à l'issue des précédentes opérations archéologiques. Si le plan général des bâtiments et leur organisation souffrent encore de nombreuses incertitudes, deux hypothèses peuvent être dégagées.

La première consisterait dans l'identification d'un vaste bâtiment à galeries reconnu sur plus $45 \mathrm{~m}$ d'est en ouest et $28 \mathrm{~m}$ du nord au sud. Abritant la dépouille de saint Martin, il aurait participé à l'organisation du culte 


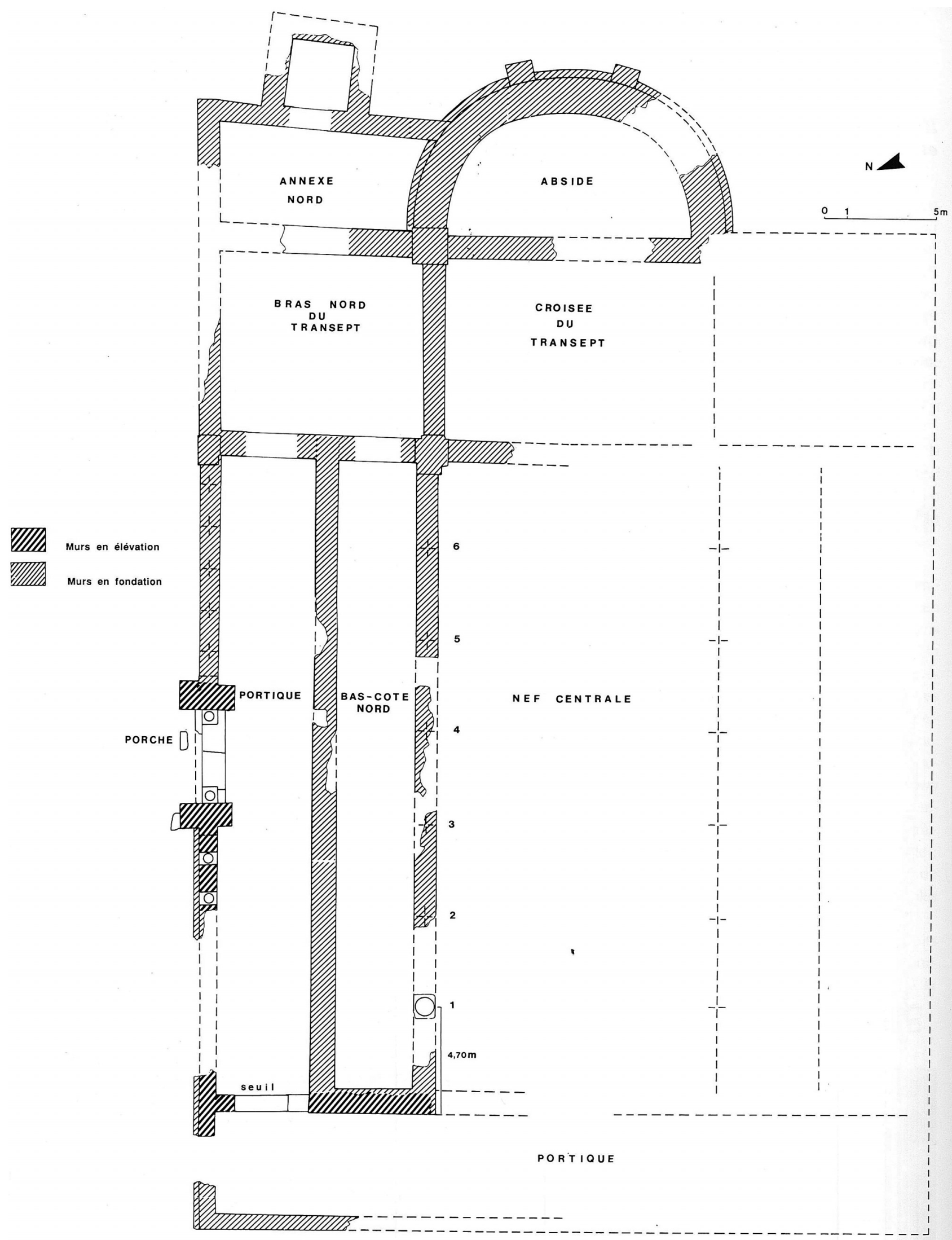

Fig. 25 : Plan d'interprétation de la basilique Saint-Laurent de Choulans à Lyon (C H. Delhumeau ds Reynaud J.-F. 1995, p. 280). 
vraisemblablement dès la fin du $\mathrm{V}^{\mathrm{e}}$ siècle. De tels dispositifs ont été reconnus pour la basilique Saint-Blaise de Saint-Mître-les Remparts (17) ou Saint-Laurent de Choulans à Lyon (fin $\mathrm{V}^{\mathrm{e}}$ siècle). Dans ce dernier cas, les proportions sont assez semblables $(49,80 \mathrm{~m}$ de longueur 19,60 m de largeur). Deux portiques ont été identifiés à l'ouest et au nord dans l'emprise de fouille limitée à la moitié nord de l'édifice (Reynaud 1995, 279-280). Un porche, situé au milieu de la galerie, garantissait un accès depuis l'extérieur (fig. 25).

L'autre hypothèse consisterait en la présence de bâtiments funéraires (oratoires, mausolées) et d'enclos progressivement développés en marge de la sépulture de Martin. Une telle organisation a notamment pu être démontrée autour de saint Seurin à Bordeaux $\left(I V^{\mathrm{e}}-\mathrm{V}^{\mathrm{e}}\right.$ siècle). La nécropole semble en effet s'organiser autour de 2 enclos délimités par des maçonneries (fig. 26, $\mathrm{n}^{\circ} 1$ et 4 ) autour desquels se sont greffés plusieurs petits mausolées $\left(n^{\circ} 7,2,3,8\right.$; Michel 2012, 287-288). Les surfaces de ces mausolées demeurent étroites (autour de $20 \mathrm{~m}^{2}$ ) et sont délimitées par des maçonneries assez frustres. Montés en petit appareil, leur largeur varie entre 0,40 et $0,60 \mathrm{~m}$. Ces différentes constructions régissent les inhumations dont l'implantation semble toutefois plus lâche en marge du site, sur la partie méridionale.

L'état des recherches ne permet pas de favoriser une quelconque hypothèse, mais l'existence de zones distinctes à l'intérieur des lieux de culte est admise et évoquée par les sources (Creissen 2009, 90), même si sa matérialisation demeure souvent complexe à définir. Quelle que soit l'option retenue, elle confirme l'état des lieux dressé au cours d'une table ronde récente concernant la diversité des caractères morphologiques de ce

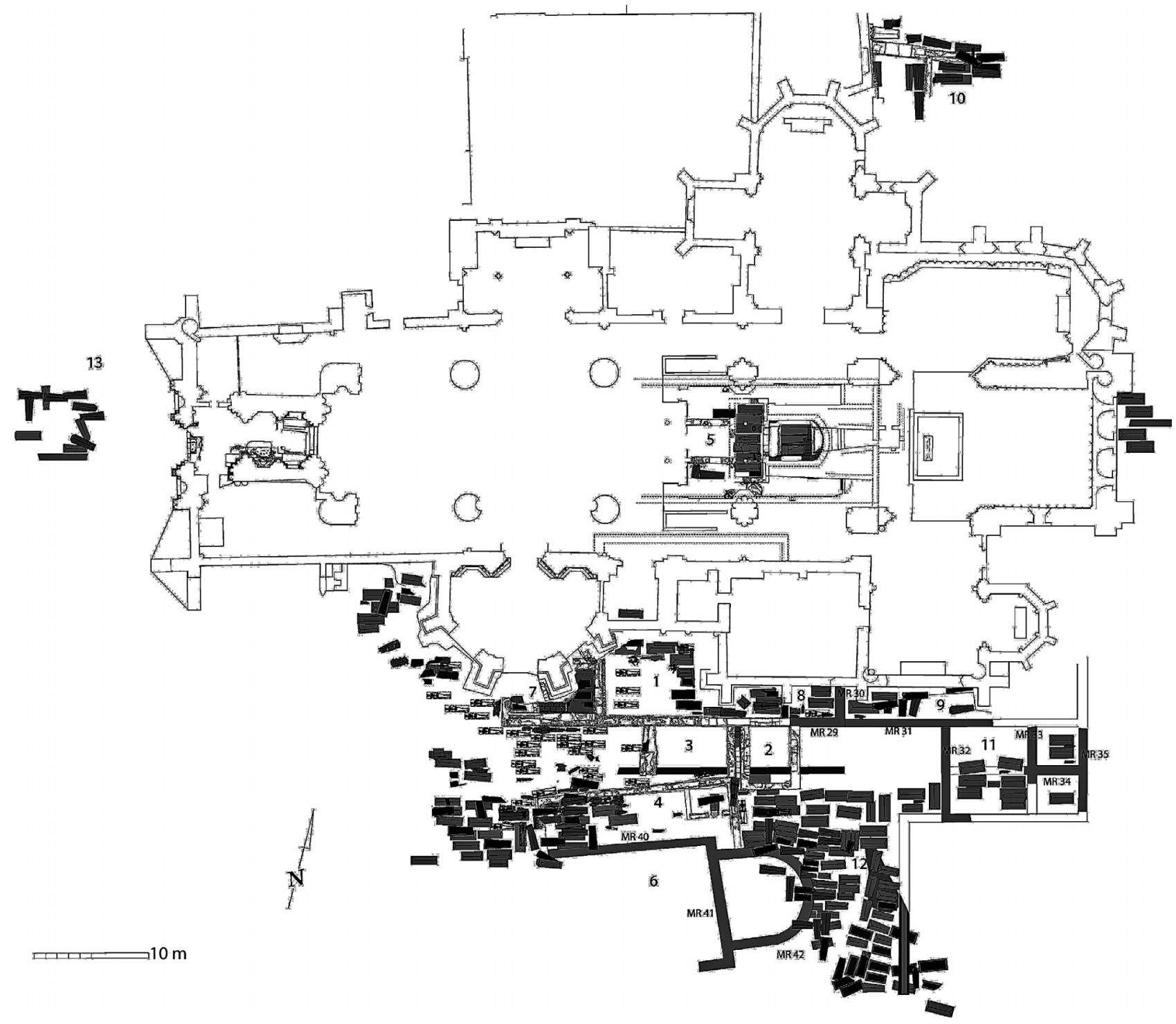

Fig. 26 : Plan d'ensemble de la nécropole Saint-Seurin (C J.-F. Pichonneau, SRA Nouvelle Aquitaine ds Michel A. 2012, p. 288).

\footnotetext{
${ }^{17}$ France, Bouches-du-Rhône
} 
type de complexe. Ce « souci de prise de possession religieuse du temps et de l'espace », se manifeste selon l'auteur par une diversité " considérable » de solutions formelles (Caillet 2015, 111).

La topographie funéraire de Brive paraît figée à la fin $\mathrm{du}$ VII ${ }^{\mathrm{e}}$ siècle. Les inhumations semblent cesser, l'espace ne retrouvant sa vocation funéraire que dans le courant $\mathrm{du} \mathrm{XI}^{\mathrm{e}}$ siècle. Un cimetière paroissial se développe autour de la collégiale et son chapitre nouvellement régularisé. Une communauté probablement chargée de l'entretien semble pourtant avoir résidé à proximité du site avant cette longue période précédant la régularisation du chapitre (vers 1100-1130) (18). Ainsi, un « abandon » de l'espace peut être exclu d'autant que le culte de Martin est reconnu pour cette période (865) au sein du martyrologue d'Usuard.

\section{SOURCES IMPRIMÉES}

Grégoire de Tours : Gregorii Turonensis Historiarum Libri X, éd. B. Krusch, Monumenta Germaniae Historica, Scriptores Rerum Merovingicarum, T. 1, Hanovre, 1885.

Historiae Francorum : Grégoire de Tours, Histoire des Francs, ed. t trad. R. Latouche, Paris, Les Belles Lettres, 2 vol, 1963.

\section{BIBLIOGRAPHIE}

Audouy 1977 : AUDOUY (M.), Sondages archéologiques autour de l'église Saint-Martin, Bulletin de la Société Scientifique, Historique et Archéologique de la Corrèze, T. 99, 1977, p. 99-110.

Barbier et al. 2015 : BARBIER (E.), LAGORSSE (K.), DUNEUFJARDIN (P.), Limousin, Corrèze, Brive-laGaillarde, Place Charles de Gaulle-Place Latreille. Nouvelles découvertes aux abords de la collégiale Saint-Martin ( $V^{e}-X I X^{e} s$.), Rapport de fouille, Inrap, 2015, 4 volumes.

Bonnay 1878 : BONNAY (L.), Description des découvertes archéologiques faites à l'église Saint-Martin de Brive, Bulletin de la Société Scientifique, Historique et Archéologique de la Corrèze, t. I, Brive, 1878, p. 223-238.

Caillet 2015 : CAILLET (J.-P.), Conclusions, in : PAIN (M.-L.), dir., Groupe cathédraux et complexes monastiques, le phénomène de la pluralité des sanctuaires à l'époque carolingienne, Presses Universitaires de Rennes, Rennes, 2015, p. 109-111.
Cantié 1988 : CANTIÉ (G.), Saint-Martin de Brive : édifices paléochrètiens et pré-romans, Travaux d'Archéologie Limousine, t. 8, Limoges, 1988, p. 67-79.

Cantié 1996 : CANTIÉ (G.), Brive-la-Gaillarde. SaintMartin. Édifice paléochrétien, Les premiers monuments chrétiens de la France, tome 2 : Sud-Ouest et Centre, Picard, Paris, p. 134-137.

Colin 2008 : COLIN (M.-G.), Christianisation et peuplement des campagnes entre Garonne et Pyrénées, IV'X siècles, Archéologie du Midi Médiéval, Supplément $n^{\circ} 5,2008$.

Creissen 2009 : CREISSEN (T.), L'aménagement du sanctuaire dans les églises de France avant l'an mil, Hortus Artium Médievalium, vol. 15, 2009, p. 87-103.

Delhoume, Roger 2013 : DELHOUME (R.), ROGER (J.), L'étude des sarcophages (analyse critique et orientations nouvelles) : une contribution à la question des cimetières du haut Moyen Âge en Creuse, Aquitania, t. 29, 2013, p. 239-287.

Massoni 2012 : MASSONI (A.), La collégiale et le chapitre Saint-Martin de Brive, de leurs origines à la Révolution, Bulletin de la Société Scientifique, Historique et Archéologique de la Corrèze, t. 134, 2012, p. 105-128.

Montigny 2011 : MONTIGNY (A.), Tulle " Place Bertaud et place Gambetta », Rapport Final d'Opération, Inrap, 2011.

Moser et al. 1986 : MOSER (F.), DAULIAT (P.), TILHARD (J.-L.), Le Puy Saint-Pierre, des origines au Moyen Âge, d'après les résultats de la fouille de la parcelle BL 432, Bulletin de la Société Scientifique, Historique et Archéologique de la Corrèze, t. 108. Brive, 1986, p. 77-102.

Reynaud 1995 : REYNAUD (J.-F.), Lyon : basilique Saint-Laurent de Choulans, in : DUVAL (N.), dir., Les premiers monuments chrétiens de la France, tome 1 : Sud-Est et Corse, Picard, Paris, 1995, p. 278-282.

Rougé 2014 : ROUGÉ (G.), Les sarcophages entre Loire et Pyrénées du $\mathrm{IV}^{e}$ au VIII siècle : observations et étude par les critères techniques et morphologiques, thèse inédite, Bordeaux, 2014.

Wyss 1996 : WYSS (M.), dir., Atlas historique de SaintDenis, des origines au XVIII ${ }^{e}$ siècle, DAF 59, Maison des sciences de l'homme, Paris, 1996.

\footnotetext{
${ }^{18}$ Massoni 2012, p. 112. L'auteure précise qu'il est très difficile, en l'absence de source, de donner une date précise pour ce changement institutionnel.
} 